\title{
An electricity market with fast bidding, planning and balancing in smart grids
}

\author{
Nicolas Höning ${ }^{a, *}$ and Han La Poutré ${ }^{a, b}$ \\ ${ }^{a}$ Centrum Wiskunde en Informatica, Amsterdam, The Netherlands. \\ ${ }^{b}$ Technical University Delft, The Netherlands.
}

January 6, 2015

\begin{abstract}
In future energy systems, peaks in the daily electricity generation and consumption are expected to increase. The "smart grid" concept aims to maintain high levels of efficiency in the energy system by establishing distributed intelligence. Software agents (operating on devices with unknown computational capabilities) can implement dynamic and autonomous decision making about energy usage and generation, e.g. in domestic households, farms or offices. To reach satisfactory levels of efficiency and reliability, it is crucial to include planning-ahead of the energy-involving activities. Market mechanisms are a promising approach for large-scale coordination problems about energy supply and demand, but existing electricity markets either do not involve planning-ahead sufficiently or require a high level of sophistication and computing power from participants, which is not suitable for smart grid settings. This paper proposes a new market mechanism for smart grids, ABEM (Ahead- and Balancing Energy Market). ABEM performs an ahead market and a last-minute balancing market, where planning-ahead in the ahead market supports both binding ahead-commitments and reserve capacities in bids (which can be submitted as price functions). These features of planningahead reflect the features in modern wholesale electricity markets. However, constructing bids in ABEM is straightforward and fast. We also provide a model of a market with the features mentioned above, which a strategic agent can use to construct a bid (e.g. in ABEM), using a decision-theoretic approach. We evaluate ABEM experimentally in various stochastic scenarios and show favourable outcomes in comparison with a benchmark mechanism.
\end{abstract}

Keywords: Markets for electricity, agent-based simulation, decision theory, smart grid

\section{Introduction}

In future energy systems, novel supply and demand patterns pose new challenges for the management and allocation of electricity. Intermittent renewable energy generators (e.g. solar panels and wind mills) increase the uncertainty on the supply side and novel consumption technologies (e.g. electric vehicles and heat pumps) increase overall demand for electricity, but also enable the demand side to become more flexible. Traditionally, demand was predictable and generation could be scheduled. The operation of an energy system could be managed by a central scheduling algorithm. In the future, however, the novel supply and demand patterns make the problem of operating a stable and affordable energy system more complicated.

Market mechanisms are a promising approach for such coordination problems. In order to increase competition and find better allocations, centrally-mediated markets for electricity, e.g. auction mechanisms, have been developed and employed on the wholesale level in recent years. A

${ }^{*}$ Corresponding author: Nicolas Höning, Intelligent Systems group, CWI Amsterdam, Science Park 123, 1098 XG Amsterdam, The Netherlands e-mail: nicolas@cwi.nl. 
new approach, often referred to as the "smart grid", aims to involve many more actors in decision making than are present on the wholesale markets. It uses a distributed intelligence approach, in which decisions are delegated to intelligent software agents on lower levels of the grid, which operate production or consumption devices on their owner's behalf (e.g. in domestic homes, farms or offices). This can be especially useful to manage flexible demand devices (e.g. heat pumps or electric vehicles).

A market mechanism suitable for smart grids should satisfy two crucial requirements. First, in order to maintain satisfactory efficiency levels in the operation of energy systems, it is important that the mechanism enables participants to plan their generation or consumption ahead of the time of actual power flow. Planning-ahead in a market mechanism (by negotiation of possible quantities and prices) facilitates planning of activities for participants and has been found to increase competition [3, 14], which lowers prices and their volatility [35]. Planning-ahead also enables flexible market participants to achieve monetary compensation for offering their flexibility. Modern wholesale electricity markets have two ways of planning-ahead - binding commitments made in an ahead market (say, 24 hours before power flow) and optional (reserve) capacity. The latter represents an option, provided by flexible participants, to supply or consume given amounts on short notice, which can be activated during a balancing phase close to the time of power flow. Such balancing is necessary since supply has to match demand due to laws of physics. However, the operation of three different allocation mechanisms, an ahead market, a market for reserve capacities and a balancing phase, introduces considerable complexity for the involved participants. This is e.g. due to the interdependence and complexity of these mechanisms.

Second, it is important to limit the complexity of bidding in the mechanism, since a smart grid setting contains many agents with limited computational capacities. For instance, heat pumps might have logic implemented on an embedded micro-chip, which they would use to decide when to buy electricity in order to heat a house or office building at the lowest cost. Or homes may have an energy management system, typically with computationally limited power. This fact prohibits to re-use market designs which have been developed on the wholesale level, where only a few big players interact who have many capabilities and much computational power to optimise their bidding behaviour, and where the aggregated supply and the aggregated demand are usually more predictable.

Most market designs in the electricity domain do not take the computational complexity of bidding into consideration sufficiently, and a smart-grid setting would not seem feasible. There have been several proposals for smart grid-inspired market mechanisms, which only require low computational effort for the creation of bids. However, these proposals either do not fully support the features of modern market mechanisms for planning-ahead or have some other trait which rules them out to be applicable as a generic mechanism for smart grids. Proposals developed in the electricity domain commonly neglect to address strategic behaviour of bidders or the convergence characteristics of their market clearing algorithm are uncertain (i.e. market outcomes can take long to be computed). Proposals developed in computer science are usually focused on specific applications, which concern sub-cases of smart grids.

There is thus a need for a generically applicable market mechanism for smart grids, which includes planning-ahead sufficiently, but which does not introduce a high computational complexity to the process of bidding. In this paper, we address this challenge by presenting ABEM, a novel market mechanism (Section 2). We also provide a decision-theoretic model for bidders in markets with the same main features as ABEM (Section 3) and perform an experimental evaluation (Section 4).

\section{The ABEM mechanism}

\subsection{Main features}

In the electricity domain, several features of market mechanisms have been developed and established in recent decades. It appears that in many countries, a distinct set of features which 
concern planning-ahead is considered to be important for efficient operation of energy systems. Our market mechanism ABEM has these important features, as well.

The first feature is a so-called two-settlement procedure [5], conducting an ahead market as well as a real-time balancing market. In this paper, we denote the ahead market (the first settlement) as "market $A$ " and the balancing market (the second settlement) as "market $B$ ". Two-settlement procedures are periodic auctions for a specific time slot, rather than continuous auctions [26]. The second feature is to also integrate the trade of reserve capacities, which are traded in the ahead market and allocated in the balancing market. The System Operator (SO) usually chooses the overall amount of required reserve capacity by experience or some heuristics (e.g. a percentage of forecasted load). The third feature is that bids for binding commitments and bids for reserve capacity have to be submitted simultaneously in the ahead market, which reduces the opportunities for strategic bidding $[8,25]$. Figure 1 shows a schematic overview of the timeline in this type of market.

\begin{tabular}{|c|c|c|}
\hline $\begin{array}{l}\text { Ahead } \\
\text { market }\end{array}$ & $\begin{array}{l}\text { Balancing } \\
\text { market }\end{array}$ & $\begin{array}{l}\text { power flow } \\
\text { time slot }\end{array}$ \\
\hline
\end{tabular}

1. All bidders submit bids for binding 4. Inflexible bidders submit bids commitments

2. Flexible bidders submit bids for reserve capacity.

3. SO allocates binding commitments and reserve capacity. for acquiring balancing power.

5. SO allocates balancing power from reserve capacity.

Figure 1: Timeline in a two-settlement procedure with integrated trade of reserve capacity and simultaneous bidding.

\subsection{Bid format}

\subsubsection{Allocation variables and the ratio between binding commitments and reserve capacity}

For each bidding agent $a$, we denote with $q_{a}^{A} \geq 0$ the binding commitment, which is allocated in market $A$ and with $q_{a}^{B} \geq 0$ the usage of reserve capacity which is allocated in market $B$. If $a$ is not flexible and thus demanding reserve capacity (in the form of upward regulation) in market $B, q_{a}^{B}<0$. If $a$ is flexible and thus offering reserve capacity, $q_{a}^{B} \in\left[0, q_{a}^{R}\right]$, where we denote with $q_{a}^{R} \geq 0$ the amount of reserve capacity which a flexible bidding agent $a$ agrees to hold.

When $a$ is a supplier, he supplies $q_{a}^{A}+q_{a}^{B}$. The maximal amount $a$ could supply is in this case $q_{a}^{\max }=q_{a}^{A}+q_{a}^{R}$, where $0 \leq q_{a}^{\max } \leq q_{a}^{U}$. On the other hand, when $a$ is a consumer, $a$ consumes $q_{a}^{A}-q_{a}^{B}$ and the maximal amount $a$ could consume is in this case $q_{a}^{\max }=q_{a}^{A}$, where $0 \leq q_{a}^{\max } \leq q_{a}^{U}$.

In the remainder of this section, we explain how the reserve capacity $q_{a}^{R}$ is defined in relation to $q_{a}^{A}$. Each agent $a$ chooses a ratio $r \in[0,1]$ per bid $b_{a, r}$. With $r$, the reserve capacity $q_{a}^{R}$ can be described as a ratio of the binding commitment $q_{a}^{A}$ and is given by:

$$
q_{a}^{R}=r q_{a}^{\max }
$$

For the case that $a$ is a supplier, we can compute $q_{a}^{R}=\frac{r q_{a}^{A}}{1-r}$ by inserting $q_{a}^{A}+q_{a}^{R}$ for $q_{a}^{\max }$ (see above). When $a$ is a consumer, this translates to $q_{a}^{R}=r q_{a}^{A}$ (because $q_{a}^{\max }=q_{a}^{A}$, see also above). Let us consider an example where $a$ submits a bid with $r=\frac{1}{3}$. For the case when $a$ is a supplier, $q_{a}^{R}=\frac{1}{3} q_{a}^{A} / \frac{2}{3}=\frac{q_{a}^{A}}{2}$. When $a$ is a consumer, then $q_{a}^{R}=\frac{q_{a}^{A}}{3}$.

At $r=0$, no reserve capacity is offered in market $B$ and $a$ has full certainty how much he sells or consumes after market $A$ has cleared $\left(q_{a}^{\max }=q_{a}^{A}, q_{a}^{R}=0\right)$. Thus, inflexible bidding agents submit bids with $r=0$ and offer no reserve capacity. For the special case $r=1$, we define that all 
of $a$ 's capacity up to $q_{a}^{\max }$ is flexible to be allocated as $q_{a}^{B}$ in market $B$. Then, if $a$ is a supplier, $q_{a}^{A}=0$ and $q_{a}^{\max }=q_{a}^{R}$, and if $a$ is a consumer, $q_{a}^{R}=q_{a}^{A}$.

\subsubsection{Bid components}

A bid in ABEM by a bidder $a$ consists of a function $b_{a, r}$ which maps marginal prices to quantities of power $\left(q_{a}^{A}\right.$ in market $A$ and $q_{a}^{B}$ in market $B$ ). The marginal value for a given quantity denotes the cost of producing the last unit or the utility of consuming the last unit. Marginal values are often used for bids in markets. They represent true information which is often accessible to the bidder, but selling or buying at marginal value nonetheless yields economic surplus for the bidder, as sellers sell all units but the last above their production costs and buyers buy all units but the last below the utility of consuming them. Another interesting economic property is that in a market with perfect competition, the marginal valuation actually represents the bid which maximises surplus [28].

It includes the ratio $r$ (see Section 2.2.1), which is unique for $b_{a, r}$. A bid in ABEM also contains lower and upper quantity limits $q_{a}^{L} \geq 0$ and $q_{a}^{U} \geq 0$, which are unique for $a$.

We restrict the function in bids to continuous linear functions. This allows for simpler optimisation during market clearing [4], but limits the ability to represent non-continuous costs like the costs of starting up or down a generator or switching from charging to discharging. Thus, ABEM is an exchange market rather than a Pool market [21].

A bid function $b_{a, r}$ in ABEM defines a positive quantity for each price $\rho \geq 0$ and is given by

$$
b_{a, r}(\rho)=\delta_{a}\left(\rho-v_{a}\right)
$$

where, if $a$ is a supplier, $v_{a}$ denotes the reservation price below which $a$ is not willing to sell and the slope parameter $\delta_{a}$ is positive. If $a$ is a consumer, $v_{a}$ denotes the reservation price above which $a$ is not willing to buy and the slope parameter $\delta_{a}$ is negative. Besides being constrained by the reservation price $v_{a}$, the set of well-defined outcomes is further constrained by quantities $q_{a}^{L}$ and $q_{a}^{U}$, so the function $b_{a, r}$ is valid only for unit prices in the interval $\left[b_{a, r}^{-1}\left(q_{a}^{L}\right), b_{a, r}^{-1}\left(q_{a}^{U}\right)\right]$.

\subsubsection{Bid translation for market $\mathrm{B}$}

After the first settlement (in market $A$ ), the SO translates each accepted bid function $b_{a, r}$ into a new bid function $b_{a}^{B}$, which is used on $a$ 's behalf in market $B . b_{a}^{B}$ is valid for unit prices in the interval $\left[\rho_{b_{a, r}}^{A}, \rho_{a, \text { max }}^{B}\right]$, where we denote with $\rho_{b_{a, r}}^{A}$ the unit price which bid $b_{a, r}$ describes for the quantity $q_{a}^{A}$, which is at the time of translation known and fixed. Thus, $\rho_{b_{a, r}}^{A}=b_{a, r}^{-1}\left(q_{a}^{A}\right)$. Furthermore, $\rho_{a, \max }^{B}=b_{a, r}^{-1}\left(q_{a}^{A}+q_{a}^{R}\right)$ if $a$ is a supplier. If $a$ is a consumer, $\rho_{a, \max }^{B}=b_{a, r}^{-1}\left(q_{a}^{A}-q_{a}^{R}\right)$.

$b_{a}^{B}$ is formulated in the same ways as $b_{a, r}$, with $v_{a}$ in Equation 2 replaced by $\rho_{b_{a, r}}^{A}$. We also introduce a second slope parameter $\omega$. When $a$ is a supplier, $\omega=1$ and if $a$ is a consumer, $\omega=-1$. Thus, if $a$ is a supplier, $b_{a}^{B}$ has the same slope as $b_{a, r}$ and if $a$ is a consumer, the slope of $b_{a}^{B}$ is inverted (with respect to the slope of $b_{a, r}$ ), because $a$ acts as a seller on market $B . b_{a}^{B}$ is given by:

$$
b_{a}^{B}(\rho)=\omega \delta_{a}\left(\rho-\rho_{b_{a, r}}^{A}\right)
$$

Figure 2 illustrates the bid translation. We note that the slope of $b_{a}^{B}$ is always positive and that the reserve price of $b_{a}^{B}$ is $\rho_{b_{a, r}}^{A}$. Thus, this translation ensures that $\rho_{a}^{B}$, the price $a$ is paid for $q_{a}^{B}$, is higher than $\rho_{a}^{A}$, the price $a$ is paid (when he is a supplier) or pays (when is a a consumer) for $q_{a}^{A}$ :

$$
\rho_{a}^{B}>\rho_{a}^{A}
$$

This reflects a relation between ahead- and balancing prices which is recommended by economic experts. For example, Oren (2000) [25] argues that balancing power is a good of higher economic quality than day-ahead procurement because of shorter delivery time and should be priced higher. 


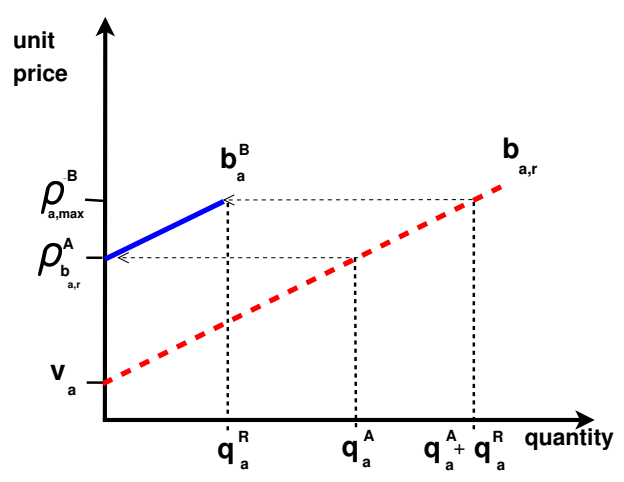

(a) $a$ is a supplier

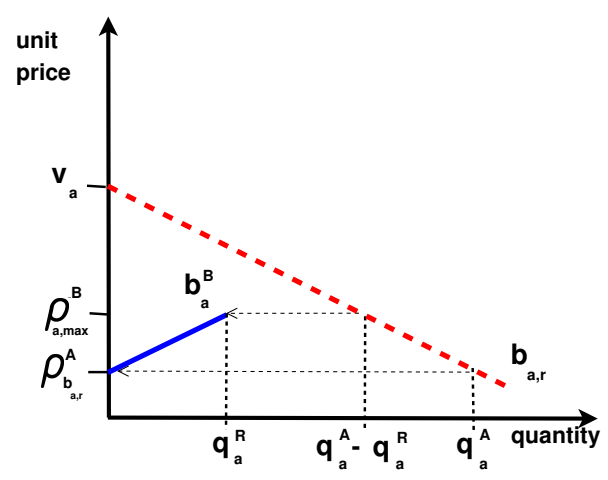

(b) $a$ is a consumer

Figure 2: Bid translation in ABEM from market $A$ (dashed) to market $B$ (continuous). The part of the bid function $b_{a, r}$ which is defined for quantities $q \in\left[q_{a}^{A}, q_{a}^{A}+q_{a}^{R}\right]$ (if $a$ is a supplier) or quantities $q \in\left[q_{a}^{A}-q_{a}^{R}, q_{a}^{A}\right]$ (if $a$ is a consumer) is translated into a new bid function $b_{a}^{B}$, which is defined for quantities $q \in\left[0, q_{a}^{R}\right]$. If $a$ is a consumer, the slope is multiplied by -1 .

\subsection{Market clearing in ABEM}

\subsubsection{Actors and bids}

We consider four sets of actors: flexible suppliers $F S$ and flexible consumers $F C$, as well as inflexible suppliers $I S$ and inflexible consumers $I C$. Bidders in $F S$ and $F C$ can provide upward regulation - they supply more or consume less, respectively, than was allocated for them as binding commitment in market $A$. They submit bids with $r \in[0,1]$. Bidders in $I S$ and $I C$ do not provide upward regulation and thus submit bids with $r=0$. However, they announce extra demand after market $A$ has cleared, because they supply less or consume more, respectively, than was allocated for them as binding commitment in market $A$. As we explained in Section 1, it is the responsibility of the SO to secure sufficient reserve capacity in market $A$, in order to supply all possible extra demand in market $B$.

For convenience, we consider in this work the case of one submitted bid per bidder. In principle, each bidder can submit more than one bid, which increases the number of market clearings that need to be performed by the SO to find the best clearing solution among all sets of choices of one bid per bidder.

\subsubsection{The constrained optimisation problem}

The optimisation goal of the SO is to solve the so-called economic dispatch problem, which is to minimise costs of electricity generation. Given all submitted bids (for each bidder $a$, the bids $b_{a, r}$ and $b_{a}^{B}$ ), the goal is to find the prices which minimise the overall objective function given by:

$$
\arg \min _{P^{A}, P^{B}}\left[\sum_{a \in F S \cup I S}\left(q_{a}^{A} \rho_{a}^{A}+q_{a}^{B} \rho_{a}^{B}\right)\right]
$$

where $P^{A}$ and $P^{B}$ denote the sets of unit prices that all bidders are allocated in market $A$ and market $B$, respectively, and $\rho_{a}^{A}$ and $\rho_{a}^{B}$ denote the prices for individual bidders $a$. It is implied that for each bidder $a, q_{a}^{A}=b_{a, r}\left(\rho_{a}^{A}\right)$ and $q_{a}^{B}=b_{a}^{B}\left(\rho_{a}^{B}\right)$.

This optimisation problem cannot be solved during the market clearing in market $A$ (the first settlement), because the bids from inflexible actors in $I S$ and $I C$, in which they describe their extra demand, are not known yet. The outcomes of market $B$ (the second settlement), namely the quantities $q_{a}^{B}$ and the prices $\rho_{a}^{B}$, can only be taken into account once these bids are known. Therefore, we break up this ex-post optimisation problem into two ex-ante optimisation problems, 
one that can be solved during the market clearing for market $A$ and another that can be solved during the market clearing for market $B$.

Market $A$ In the optimisation problem for the market clearing in market $A$ (for binding commitments and reserve capacity), the SO minimises the costs which are known for sure at the time of this market clearing and, to an extent which the SO chooses, the costs he expects to occur in the market clearing of market $B$. This optimisation problem is given by:

$$
\arg \min _{P^{A}}\left[\sum_{a \in F S \cup I S} q_{a}^{A} \rho_{a}^{A}+\gamma E\left[C^{B}\right]\right]
$$

where $E[X]$ denotes the expectation of $X, C^{B}$ denotes costs of using reserve capacity in market $B\left(C^{B}=\sum_{a}^{F S \cup F C} q_{a}^{B} \rho_{a}^{B}\right)$ and $\gamma \in[0,1]$ is a weight parameter which the SO can choose. By estimating $C^{B}$, the SO estimates costs in market $B$, but does not include the price set $P^{B}$ as optimisation variables.

If the SO chooses $\gamma=0$, there is no need to estimate $C^{B}$ and the outcomes of market $B$ are not considered during the clearing in market $A$. If he chooses $\gamma>0$ and also estimates $C^{B}$ close to the actual $C^{B}$, the $\mathrm{SO}$ can improve the solution to the overall economic dispatch problem in Equation 5 by buying more power on market $A$ than inflexible consumers ordered, in the expectation that some inflexible actors will have to order more expensive balancing power in market $B$. This can reduce overall costs because it holds for each flexible actor $a$ that $\rho_{a}^{B}>\rho_{a}^{A}$ (see Section 2.2.3).

We now list the constraints that every valid solution needs to respect. First, supply needs to equal demand:

$$
\sum_{a \in I S}\left(q_{a}^{A}+E\left[q_{a}^{B}\right]\right)+\sum_{a \in F S}\left(q_{a}^{A}+q_{a}^{B}\right)=\sum_{a \in I C}\left(q_{a}^{A}+E\left[q_{a}^{B}\right]\right)+\sum_{a \in F C}\left(q_{a}^{A}-q_{a}^{B}\right)
$$

If the SO chooses $\gamma=0$, the SO does not need to consider (expectations of) $q_{a}^{B}$ and this constraint can be simplified to:

$$
\sum_{a \in F S \cup I S} q_{a}^{A}=\sum_{a \in F C \cup I C} q_{a}^{A}
$$

Furthermore, the SO needs to make sure that each supplier $a$ will stay within his capacity constraints:

$$
q_{a}^{L} \leq q_{a}^{A} \leq q_{a}^{U}\left(1-r_{a}\right)
$$

where $r_{a}$ is the ratio between binding commitment and reserve capacity (see Section 2.2.1) from $a$ 's bid. Similarly, each consumer $a$ needs to stay within his capacity constraints:

$$
q_{a}^{L}+q_{a}^{A} r_{a} \leq q_{a}^{A} \leq q_{a}^{U}
$$

Flexible suppliers and consumers are allocated reserve capacity $q_{a}^{R}$, as described in Section 2.2.1. The overall reserve capacity needs to be at least as high as $q^{R}$, the overall reserve capacity, which is determined by the $\mathrm{SO}$ (see Section 1). Hence, we add the final constraint

$$
\sum_{a \in F S \cup F C} q_{a}^{R} \geq q^{R}
$$

We could have used $=$ instead of $\geq$, but this is not necessary, as the cost optimisation is ensured by minimising costs of supply. 
Market $B$ In the optimisation problem for the market clearing in market $B$, the $\mathrm{SO}$ minimises the costs for the use of reserve capacity. Before market $B$ is cleared, inflexible actors $a \in I S \cup I C$ announce their extra demand $q_{a}^{B}$ (flexible actors do not need to do that). The $S O$ translates each accepted bid $b_{a, r}$ from flexible actors (submitted during the first settlement in market $A$ ) into a bid $b_{a}^{B}$ (to be used in market $B$ ), as described in Section 2.2.3. These translated bids are used to minimise the objective function given by

$$
\arg \min _{P^{B}}\left[\sum_{a \in F S \cup F C} q_{a}^{B} \rho_{a}^{B}\right]
$$

The only constraint to this optimisation requires that all supply equals all demand:

$$
\sum_{a \in I S}\left(q_{a}^{A}-q_{a}^{B}\right)+\sum_{a \in F S}\left(q_{a}^{A}+q_{a}^{B}\right)=\sum_{a \in I C}\left(q_{a}^{A}+q_{a}^{B}\right)+\sum_{a \in F C}\left(q_{a}^{A}-q_{a}^{B}\right)
$$

\subsubsection{Uniform and discriminative pricing}

There exist two major approaches to find prices from bids in auctions - uniform pricing (UPA) and discriminative pricing (DPA) [17]. If a UPA design approach to market clearing is used, then the price sets $P^{A}$ and $P^{B}$ contain the same prices $\rho_{a}^{A}$ and $\rho_{a}^{B}$ for all actors $a$. In each of the two markets, the $S O$ adds up (with respect to quantities) all supply functions to one aggregated supply function $S$. In market $A$, these are the functions $b_{a, r}$ per bidder $a \in F S \cup I S$ and in market $B$, these are the functions $b_{a}^{B}$ per bidder $a \in F S \cup F C$. The SO also adds up all demand functions to one aggregated demand function $D$. In market $A$, these are the functions $b_{a, r}$ per bidder $a \in F C \cup I C$ and in market $B$, these are the functions $b_{a}^{B}$ per bidder $a \in I S \cup I C$.

In both markets, the price $\rho$ for which $S(\rho)=D(\rho)$ is the uniform clearing price. Each actor $a$ buys or sells the quantity which can be looked up on his relevant bid function $\left(b_{a, r}\right.$ or $b_{a}^{B}$, see above) at price $\rho$. Should that quantity be lower than $0, a$ sells nothing. Should that quantity be higher than a maximal limit $q_{a}^{\max }$ for this bid, $a$ sells $q_{a}^{\max }$ at price $\rho$.

Sandholm and Suri (2002) [29] showed that finding $\rho$ is not computationally expensive and always possible, under two conditions. First, all supply functions need to be monotonically increasing and all demand bids need to be monotonically decreasing, which is a condition that the bid functions we describe in Section 2.2 fulfil. Second, the bid functions should either be linear or piecewise linear. We deal with linear functions, so this condition is fulfilled, as well (capacity constraints like we described in Constraints 9 and 10 are also used in [29]). However, Constraint 11 may render the solution at price $\rho$ invalid. The SO can request that actors with flexibility submit at least one bid with the value for $r$ larger than some minimal $r_{m}$ which the SO chooses.

Finding prices in a DPA approach is computationally more elaborate, as there is now a distinct price per bidder $a$ in both $P^{A}$ and $P^{B}$. However, in [29] it is also shown that the problem of finding optimal discriminatory prices in a two-sided auction with both supply and demand curves for multiple indistinguishable units can be formulated as a convex quadratic program with linear constraints. The solution to such a program can be found in polynomial time using general techniques, e.g. with the technique described in [7]. The only condition is that curves are linear, which is given in our context, see Section 2.2.2. All the constraints we formulated in Section 2.3.2 are linear. As described for the UPA clearing, Constraint 11 can make some solutions invalid and the SO might need to request that some bids with minimal values of $r$ are submitted.

\subsection{Advantages of ABEM by design}

This section describes three advantages which ABEM has by design. Later, Section 4 also describes advantages \& disadvantages experimentally.

The complexity of bid construction is reduced. In the ABEM mechanism, bidders only submit one bid, whereas other comparable mechanisms require the submission of two separate bids, one for binding commitments and one for reserve capacity (refer to Section 1). This is 
made possible by the bid translation, described in Section 2.2.3. We will show concrete examples of the bidder's optimisation problem in our decision-theoretic experiments later on, where the simplification of the objective function becomes apparent formally.

Bidders can bid price functions to both markets, potentially their marginal costs or valuation. In $A B E M$, the function which is submitted to market $A$ is resubmitted to market $B$. The knowledge of the allocation in market $A$ is used in the translation process (see Section 2.2.3). This means that a price function is used in both markets, rather than a constant price in market $B$ (which is the case in some real-world versions of comparable mechanisms).

Being able to submit only one price function also enables bidders to submit their marginal cost or utility function as bid, which is not feasible in mechanisms which require the submission of two independent bids. The expression of the valuation is of course limited by our formal definition of price functions in Section 2.2.2. For example, costs of ramping up or down and switching costs can not be expressed, which has little effect on some flexible technologies (e.g. batteries) and more effect on others (e.g. coal power plants).

Flexible consumers are guaranteed that offering reserve capacity increases their overall utility. Flexible actors should be incentivised to offer reserve capacity. We will show by proof that the following proposition holds:

Proposition 2.1. For a flexible consumer, offering reserve capacity is guaranteed to be profitable, if he submits his marginal utility function.

As a benchmark, we consider $a$ as an inflexible consumer, who buys $q_{a}^{A}$ and does not sell anything on market $B$. His surplus $S U R_{a}^{\prime}$ is given by:

$$
S U R_{a}^{\prime}=\int_{q=0}^{q_{a}^{A}}\left(\rho_{a}(q)-\rho_{a}^{A}\right) d q
$$

where $\rho_{a}(q)=b_{a, r}^{-1}(q)$ denotes the unit price at which $a$ 's bid $b_{a, r}$ describes the quantity $q$.

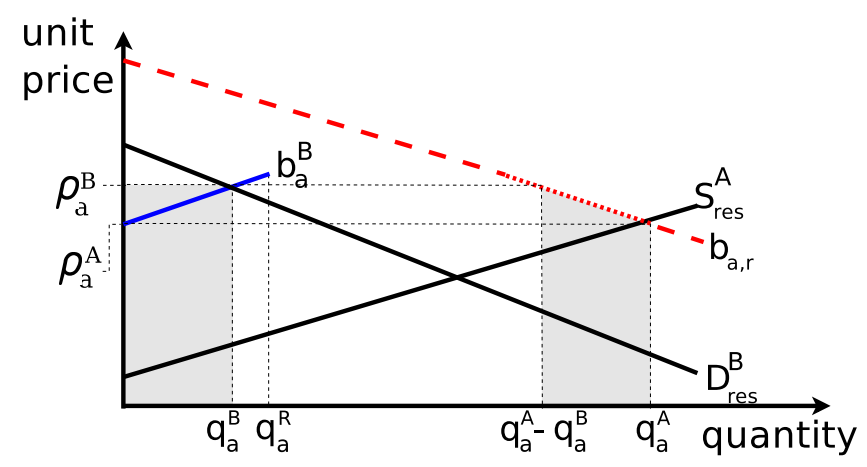

Figure 3: $a$ 's bid $b_{a, r}$ and the residual functions $S_{r e s}^{A}$ and $D_{r e s}^{B}$. The dotted part of bid $b_{a}$ is translated into bid $b_{a}^{B} . q_{a}^{A}$ and $q_{a}^{B}$ are determined by intersection of $b_{a, r}$ with $S_{\text {res }}^{A}$ and $D_{\text {res }}^{B}$.

Now we consider the case in which $a$ is flexible and active on market $B$, selling any $q_{a}^{B} \in\left[0, q_{a}^{R}\right]$ at price $\rho_{a}^{B}$. Let us denote $a$ 's utility in this case by $U_{a}^{\prime}$. We calculate $U_{a}^{\prime}$ by subtracting from $S U R_{a}^{\prime}$ the loss of utility for selling $q_{a}^{B}$ less than $q_{a}^{A}$ and adding the revenues from selling $q_{a}^{B}$. For illustration, Figure 3 shows in a grey area both the lost utility (on the right) and the revenues from selling $q_{a}^{B}$ (on the left). $U_{a}^{\prime}$ is given by: 


$$
\begin{aligned}
U_{a}^{\prime} & =S U R_{a}^{\prime}-\int_{q=q_{a}^{A}-q_{a}^{B}}^{q_{a}^{A}} \rho_{a}(q) d q+q_{a}^{B} \rho_{a}^{B} \\
& =S U R_{a}^{\prime}+\int_{q=0}^{q_{a}^{B}}\left(-\rho_{a}\left(q+q_{a}^{A}-q_{a}^{B}\right)+\rho_{a}^{B}\right) d q
\end{aligned}
$$

$U_{a}^{\prime}$ is guaranteed to be larger than $S U R_{a}^{\prime}$, if we can show that $\rho_{a}\left(q+q_{a}^{A}-q_{a}^{B}\right) \leq \rho_{a}^{B}$ for all $q \in\left[0, q_{a}^{B}\right]$. This is the case, because $\rho_{a}\left(0+q_{a}^{A}-q_{a}^{B}\right)=\rho_{a}^{B}$ and the slope of $b_{a, r}$ is negative.

Finally, we can now show that the ABEM bid mechanism guarantees that offering reserve capacity increases $a$ 's utility, compared with an inflexible consumer that buys the same amount of electricity. As a benchmark, let us again assume that $a$ is inflexible and offers nothing to market $B$ (and thus $q_{a}^{R}=0$ ). Here, we assume that $a$ buys exactly $q_{a}^{A}-q_{a}^{B}$ in market $A$. We denote the surplus for $a$ in this case by $S U R_{a}^{*}$, given by:

$$
S U R_{a}^{*}=\int_{q=0}^{q_{a}^{A}-q_{a}^{B}}\left(\rho_{a}(q)-\rho_{a}^{B}\right) d q
$$

where in this example, $\rho_{a}^{B}=\rho_{a}\left(q_{a}^{A}-q_{a}^{B}\right)$ denotes the price $a$ pays for $q_{a}^{A}-q_{a}^{B}$ on market $A$ (refer also to Figure 3).

Let us now consider that $a$ acts as a flexible consumer and offers $q_{a}^{R}$ on market $B$. To make this case comparable to our benchmark case (which led to $S U R_{a}^{*}$ ), we assume that $a$ first buys $q_{a}^{A}$ and then sells $q_{a}^{B} \in\left[0, q_{a}^{R}\right]$. This leaves $a$ with $q_{a}^{A}-q_{a}^{B}$ for his own usage, just as in the benchmark case. We denote the utility $a$ has in this case with $U_{a}^{*}$. There are two differences in $U_{a}^{*}$ with respect to $S U R_{a}^{*}$ : First, $a$ pays a lower unit price for his consumption of $q_{a}^{A}-q_{a}^{B}$, namely $\rho_{a}^{A}$ instead of $\rho_{a}^{B}$, and if the price difference of $\rho_{a}^{B}-\rho_{a}^{A}$ is positive, $a$ 's utility will increase. Second, $a$ sells $q_{a}^{B}$ instead of consuming it himself, and thus adds $U_{a}^{\prime}-S U R_{a}^{\prime}$ to his utility (see above). $U_{a}^{*}$ is given by:

$$
U_{a}^{*}=S U R_{a}^{*}+\left(\rho_{a}^{B}-\rho_{a}^{A}\right)\left(q_{a}^{A}-q_{a}^{B}\right)+U_{a}^{\prime}-S U R_{a}^{\prime}
$$

We have shown above that $U_{a}^{\prime}-S U R_{a}^{\prime}$ is positive. We now show that also $\left(\rho_{a}^{B}-\rho_{a}^{A}\right)\left(q_{a}^{A}-q_{a}^{B}\right)$ is positive. This is the case because $q_{a}^{A}-q_{a}^{B} \geq 0$ and $\rho_{a}^{B} \geq \rho_{a}^{A}$ (see Section 2.2.3).

The intuition behind this proof is that buying $q_{a}^{A}$ and then selling $q_{a}^{B}$ is better for $A$ 's utility than both only buying $q_{a}^{A}$ and only buying $q_{a}^{A}-q_{a}^{B}$ in market $A$. The first reason for this is that the more $a$ buys in market $A$, the more the price per unit bought decreases (because the slope of $b_{a, r}$ is decreasing). The second reason is that it is profitable to resell a unit in market $B$ which was bought in market $A$, because $\rho_{a}^{B}>\rho_{a}^{A}$, a property of ABEM we established in Section 2.2.3.

Proposition 2.1 is an important baseline result, especially for markets with high levels of competition. However, we are not able to make a similar claim about a flexible supplier. Let us assume that $a$ has no choice which bid function $b_{a, r}$ to submit (i.e. submitting his marginal costs is one possible scenario given this assumption). Then, $a$ prefers to sell a quantity $q$ on market $A$ over selling $q$ partly on market $A$ and partly on market $B$. Let $\rho_{a}^{A}$ denote the price $a$ is paid for $q_{a}^{A}$ $\left(b_{a, r}\left(\rho_{a}^{A}\right)=q_{a}^{A}\right)$ and let $\rho_{a}^{B}$ denote the price $a$ is paid for $q_{a}^{B}$ in market $B\left(b_{a}^{B}\left(\rho_{a}^{B}\right)=q_{a}^{B}\right)$. Given the way bids in ABEM are translated between market $A$ and market $B, \rho_{a}^{B}$ is also the price $a$ would be paid in the case where he sells all of $q_{a}^{A}+q_{a}^{B}$ already in market $\left.A\left(b_{a, r}\left(\rho_{a}^{B}\right)=q_{a}^{A}+q_{a}^{B}\right)\right)$. The difference in $a$ 's profits if he sells either $q_{a}^{A}+q_{a}^{B}$ on market $A$ or if he first sells $q_{a}^{A}$ on market $A$ and then $q_{a}^{B}$ on market $B$ is $q_{a}^{A}\left(\rho_{a}^{B}-\rho_{a}^{A}\right)$. Because $\rho_{a}^{B}>\rho_{a}^{A}$ (see above), $a$ clearly prefers the first option.

This shows that flexible suppliers cannot be guaranteed that offering reserve capacity increases their profits. Offering reserve capacity can, however, be profitable in many market settings and $a$ will have to consider this possibility when constructing his bid. 


\section{A parametrised market model for decision-theoretic bid optimisation}

\subsection{Aggregation of other actors}

The bidding behaviour of all other market participants besides $a$ is modelled as parametrised functions. For brevity of this market model, these functions are aggregated on both demand and supply side. Aggregating actors in this way is based on the assumption that the average behaviour is sufficiently predictable. Good predictions can be made either when the number of actors is high or individual decision-making of a smaller group of actors can be estimated (for instance by experience).

Following [27], an aggregated bid function is the sum of curves of individual bid functions. Let $D(\rho) \rightarrow \mathbb{R}$ be an aggregated demand function and $S(\rho) \rightarrow \mathbb{R}$ an aggregated supply function for unit prices $\rho$. We will use $D, S$ and their parameters with the superscripts ${ }^{A}$ for market $A$ and ${ }^{B}$ for market $B$. If needed for clarification, we might use the subscript ${ }_{-a}$ to denote explicitly that the function does not include $a$. $D$ and $S$ for markets $A$ and $B$ are given by

$$
\begin{aligned}
D^{A}(\rho) & :=\left[D_{\text {max }}^{A}-\alpha^{A} \rho\right]_{\geq 0} \\
S^{A}(\rho) & :=\left[\beta^{A}\left(\rho-\rho_{\text {min }}^{A}\right)\right]_{\geq 0} \\
D^{B}(\rho) & :=\left[D_{\text {max }}^{B}-\alpha^{B} \rho\right]_{\geq 0} \\
S^{B}(\rho) & :=\left[\beta^{B}\left(\rho-\rho_{\text {min }}^{B}\right)\right]_{\geq 0}
\end{aligned}
$$

where $[X]_{\geq 0}$ denotes the maximum of $X$ and $0, D_{\max }^{A}, D_{\max }^{B}$ are constants denoting the maximal demand, $\rho_{\text {min }}^{A}, \rho_{\text {min }}^{B}$ are constants denoting the minimal unit offer price and $\alpha^{A}, \alpha^{B}$ as well as $\beta^{A}, \beta^{B} \in[0,1]$ are slope parameters.

We thus have eight parameters to describe this market model. Some relations between parameters, however, might be assumed. For example, $D_{\max }^{B}$ is probably related to $D_{\max }^{A}, \rho_{\min }^{B}$ is probably not lower than $\rho_{\min }^{A}$ and the slopes of these accumulated functions can probably assumed not to change significantly between market $A$ and market $B$. We will make specific assumptions for such relations when we make use of this market model in experiments.

\subsection{Residual functions}

Given $D^{A}, D^{B}, S^{A}$ and $S^{B}$, we model the residual functions that $a$ faces in markets $A$ and $B$. In economic theory, residual supply is the full market supply minus the quantity bought by other actors at each unit price $\rho$ and residual demand is the full market demand minus the quantity supplied by other actors at each unit price $\rho$. Following [27], Equation 20 first shows the residual demand function $D_{\text {res }}^{A}$ (for when $a$ is a supplier) and then the residual supply function $S_{\text {res }}^{A}$ (for when $a$ is a consumer). Finally, the residual demand function $D_{\text {res }}^{B}$ is shown, which $a$ faces in market $B$.

$$
\begin{aligned}
D_{\text {res }}^{A}(\rho) & =D^{A}(\rho)-S_{-a}^{A}(\rho) \\
S_{\text {res }}^{A}(\rho) & =S^{A}(\rho)-D_{-a}^{A}(\rho) \\
D_{\text {res }}^{B}(\rho) & =D^{B}(\rho)-S_{-a}^{B}(\rho)
\end{aligned}
$$


Table 1: Summary of market parameters - we use superscripts ${ }^{A}$ or ${ }^{B}$ to denote usage in market $A$ or $B$, respectively.

\begin{tabular}{cl}
\hline Parameter & Description \\
\hline$D_{\max }$ & maximal demand of demand functions $D_{-a}^{A}$ and $D^{B}$ \\
$\alpha$ & slope of demand functions \\
$\rho_{\min }$ & min. price of supply functions $S_{-a}^{A}$ and $S_{-a}^{B}$ \\
$\beta$ & slope of supply functions \\
$k$ & noise parameter \\
\hline
\end{tabular}

\subsection{Market clearing}

We can now discuss how supply and demand bids are cleared in our decision-theoretic market model representation. The prices $\rho_{a}^{A}$ and $\rho_{a}^{B}$, which allocate from $a$ the quantities $q_{a}^{A}$ and $q_{a}^{B}$, respectively, are found at the intersection of $a$ 's bid with the residual functions. Similar to Equation 20, Equation 21 first shows clearing in market $A$, for the two cases of $a$ being a supplier or a consumer, and then clearing in market $B$, where $a$ acts as a supplier:

$$
\begin{aligned}
& q_{a}^{A}=D_{\text {res }}^{A}\left(\rho_{a}^{A}\right)=b_{a, r}\left(\rho_{a}^{A}\right) \\
& q_{a}^{A}=S_{\text {res }}^{A}\left(\rho_{a}^{A}\right)=b_{a, r}\left(\rho_{a}^{A}\right) \\
& q_{a}^{B}=D_{\text {res }}^{B}\left(\rho_{a}^{B}\right)=b_{a}^{B}\left(\rho_{a}^{B}\right)
\end{aligned}
$$

\subsection{Uncertainty}

$a$ approximates the residual supply and demand functions $D_{\text {res }}^{A}$ (if $a$ is a supplier), $S_{\text {res }}^{A}$ (if $a$ is a consumer) and $D_{\text {res }}^{B}$ (in both cases) with some uncertainty. We model this by noise parameters $k^{A}$ and $k^{B}$, with which we multiply the minimal price of suppliers in $S_{-a}^{A}$ and $S_{-a}^{B}$ (refer to Equations (18) and (19)). Functions $D_{\text {res }}^{A}, S_{\text {res }}^{A}$ and $S_{-a}^{A}$ prescribe an additional parameter $k^{A}$ and functions $D_{r e s}^{B}$ and $S_{-a}^{B}$ prescribe an additional parameter $k^{B} . S_{-a}^{A}$ and $S_{-a}^{B}$ are then given by:

$$
\begin{aligned}
& S_{-a}^{A}\left(\rho, k^{A}\right)=\beta^{A}\left(\rho-\rho_{\text {min }}^{A} k^{A}\right) \\
& S_{-a}^{B}\left(\rho, k^{B}\right)=\beta^{B}\left(\rho-\rho_{\text {min }}^{B} k^{B}\right)
\end{aligned}
$$

For the likelihood of individual value of $k^{A}$ and $k^{B}, a$ needs to model two probability distributions $\operatorname{prob}^{A}: \mathbb{R} \rightarrow[0,1]$ and $\operatorname{prob}^{B}: \mathbb{R} \rightarrow[0,1]$, respectively.

\subsection{Surplus functions for a}

We now model surplus functions for $a$, given market outcomes, which are useful in the economic analysis of bidding strategies in multi-unit commodity markets with unit prices (like ABEM). This work assumes that the marginal cost function of a supplier is monotonically increasing and that the marginal utility function of consumers is monotonically decreasing. This follows from the economic assumption that those units which cost the least to produce are produced first and that each consumed unit will increase utility less than the one which was consumed before it. We consider only variable costs and do not model fixed costs explicitly. This leads us to model producer and consumer surplus (which we define below) instead of economic, long-term profit.

Producer's surplus is defined as the revenue that a supplier receives for his delivered quantity minus the variable costs of producing it (where revenue is defined as quantity times unit price). Consumer's surplus is defined as the utility derived from consuming a quantity of goods (which is the highest value that the consumer is willing to pay) minus the price he actually paid for it.

Let a bidding agent $a$ represent his total costs of generating a quantity $q$ of electricity or, alternatively, his total utility of consuming a quantity $q$ of electricity, by a quadratic valuation 
function $V_{a}$, e.g. as advocated for in [4] and modelled in [32]. $V_{a}$ and the marginal valuation function $V_{a}^{\prime}$ (the derivative of $V_{a}$ ) are given by

$$
\begin{aligned}
& V_{a}(q)=v_{a} q+\delta_{a} q^{2} \\
& V_{a}^{\prime}(q)=v_{a}+2 \delta_{a} q
\end{aligned}
$$

where $v_{a} \in \mathbb{R}$ and $\delta_{a} \in \mathbb{R}$ are coefficients. Furthermore, $\delta>0$ for cost functions and $\delta<0$ for consumption utility functions. $v_{a}$ denotes the value of the first unit in $q$ and $\delta_{a}$ denotes (half of) the change in value of every further unit produced or consumed.

$a$ as a flexible supplier: In market $A$, revenues are $q_{a}^{A} * \rho_{a}^{A}$ and the total costs of producing $q_{a}^{A}$ are given by $V_{a}\left(q_{a}^{A}\right)$. In market $B$, revenues are $q_{a}^{B} * \rho_{a}^{A}$ and the total costs of generating $q_{a}^{B}$ are the costs for generating the last $q_{a}^{B}$ units in $q_{a}^{A}+q_{a}^{B}$. Therefore, we introduce a total cost function $V_{a}^{B}$ for $q_{a}^{B}$ that calculates the costs on $V_{a}\left(q_{a}^{A}+q_{a}^{B}\right)$ for $q_{a}^{B} \in\left[0, q_{a}^{R}\right] . V_{a}^{B}$ is given by:

$$
\begin{aligned}
V_{a}^{B}\left(q_{a}^{A}, q_{a}^{B}\right) & =V_{a}\left(q_{a}^{A}+q_{a}^{B}\right)-V_{a}\left(q_{a}^{A}\right) \\
& =\left(v_{a}+2 \delta_{a} q_{a}^{A}\right) q_{a}^{B}+\delta_{a}\left(q_{a}^{B}\right)^{2}
\end{aligned}
$$

Then, the surplus functions are given by:

$$
\begin{aligned}
\operatorname{surplus}_{a}^{A}\left(b_{a, r}, k^{A}\right) & =\rho_{a}^{A} q_{a}^{A}-V_{a}\left(q_{a}^{A}\right) \\
\operatorname{surplus}_{a}^{B}\left(b_{a}^{B}, b_{a, r}, k^{B}\right) & =\rho_{a}^{B} q_{a}^{B}-V_{a}^{B}\left(q_{a}^{A}, q_{a}^{B}\right)
\end{aligned}
$$

where $b_{a}^{B}$ is either the result of the translation of bid function $b_{a, r}$ for market $B$ in the ABEM mechanism (see Section 2.2.3) or the price $\rho_{a}^{B}$, chosen by $a$ in the BENCH mechanism (see Section 4.1.1). $q_{a}^{A}$ and $\rho_{a}^{A}$, as well as $q_{a}^{B}$ and $\rho_{a}^{B}$, are determined through market clearing (see Section 2.3), and thus $b_{a, r}$ and $k^{A}$, as well as $b_{a}^{B}$ and $k^{B}$, are implicit in the right-hand formulae. Note that surplus $s_{a}^{B}$ is coupled to the results of market $A$ (and thus needs to consider $b_{a, r}$ ), as $q_{a}^{A}$ is used in $V_{a}^{B}$ as well as in the determination of $q_{a}^{R}$.

$a$ as a flexible consumer: $a$ 's overall utility $U_{a}$ is given by:

$$
U_{a}=V_{a}\left(q_{a}^{A}-q_{a}^{B}\right)-q_{a}^{A} \rho_{a}^{A}+q_{a}^{B} \rho_{a}^{B}
$$

For surplus $a_{a}^{A}$, we consider $a$ 's valuation of consuming $q_{a}^{A}$ and the costs of buying $q_{a}^{A}$. For surplus ${ }_{a}^{B}$, we consider the economic reward for reducing demand and subtract the costs of $a$ 's provision of reserve capacity by the (lost) utility of the last $q_{a}^{B}$ units in $q_{a}^{A}$. We model this lost utility via the function $V_{a}^{B}$, which is given by:

$$
V_{a}^{B}\left(q_{a}^{B}, q_{a}^{A}\right)=V_{a}\left(q_{a}^{A}\right)-V_{a}\left(q_{a}^{A}-q_{a}^{B}\right)
$$

Then, the surplus functions are given by:

$$
\begin{aligned}
\operatorname{surplus}_{a}^{A}\left(b_{a, r}, k^{A}\right) & =V_{a}\left(q_{a}^{A}\right)-q_{a}^{A} \rho_{a}^{A} \\
\operatorname{surplus}_{a}^{B}\left(b_{a}^{B}, b_{a, r}, k^{B}\right) & =q_{a}^{B} \rho_{a}^{B}-V_{a}^{B}\left(q_{a}^{B}, q_{a}^{A}\right)
\end{aligned}
$$

\subsection{The bid optimisation problem}

The surplus maximisation problem for $a$ is given by:

$$
\begin{aligned}
\arg \max _{b_{a, r}, b_{a}^{B}} & {\left[\int _ { k ^ { A } = k _ { \text { min } } ^ { A } } ^ { k _ { \operatorname { m a x } } ^ { A } } \operatorname { p r o b } ^ { A } ( k ^ { A } ) * \left(\operatorname{surplus}_{a}^{A}\left(b_{a, r}, k^{A}\right)\right.\right.} \\
& \left.\left.+\int_{k^{B}=k_{\text {min }}^{B}}^{k_{\text {max }}^{B}} \operatorname{prob}^{B}\left(k^{B}\right) * \operatorname{surplu} s_{a}^{B}\left(b_{a}^{B}, b_{a, r}, k^{B}\right) d k^{B}\right) d k^{A}\right]
\end{aligned}
$$


Note that in the ABEM mechanism, $b_{a}^{B}$ is not a choice to be made by $a$, but is translated from $b_{a, r}$ and therefore the optimisation problem is considerably reduced in complexity, when compared to a mechanism where bids $b_{a, r}$ and $b_{a}^{B}$ are independent.

\section{Experiments}

\subsection{Experiment models}

\subsubsection{The benchmark mechanism BENCH}

We model a benchmark mechanism (which we will refer to as BENCH from now on) for $a$ to take part in, which is modelled in resemblance to existing real-world implementations of electricity markets (e.g. [22] or [33]). Like ABEM, the BENCH mechanism is a two-settlement mechanism with integration of the trade of reserve capacity and simultaneous bidding. Unlike ABEM, BENCH requires from $a$ two separate bids to market $A$ and market $B$.

Furthermore, BENCH also requires from $a$ a price function $b_{a, r}$ as bid to market $A$ (like ABEM), but only allows $b_{a}^{B}$, the bid to market $B$ to represent a constant price $\rho_{a}^{B}$ (unlike ABEM). In both mechanisms, the reserve capacity $q_{a}^{R}$ is determined by a bid parameter, and the allocation in market $A$, as described in Section 2.2.1. Figure 4 illustrates the bids to BENCH for both cases ( $a$ representing a supplier or a consumer).

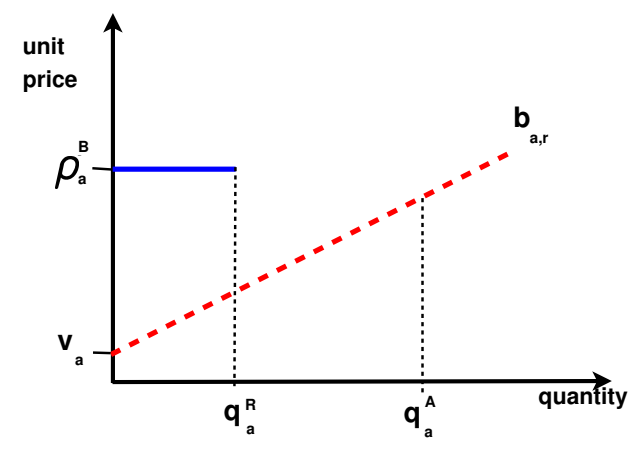

(a) $a$ is a supplier

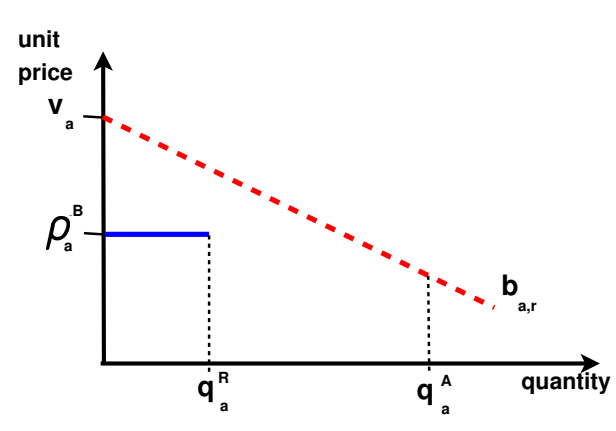

(b) $a$ is a consumer

Figure 4: Bids in the BENCH format. Bid $b_{a, r}$ to market $A$ (dashed) is a function and the bid $\rho_{a}^{B}$ to market $B$ (continuous) is a constant price. Note that $\rho_{a}^{B}$ is independent from the bid $b_{a, r}$, besides being constrained in quantity by $q_{a}^{R}$. For bids in ABEM, see Figure 2 .

Both the ABEM and the BENCH mechanism can be used for $a$ within the market model described in Section 3, without changes to the modelling of other actors.

\subsubsection{Translation of $a$ 's valuation of electricity into bids}

We now show how the bidder agent $a$ translates $V_{a}^{\prime}$, his marginal value function for electricity (refer to Section 2.2.2) into a bid function to either the ABEM or BENCH mechanism. We also show how $a$ can alter this bid function for surplus optimisation.

Bid functions in both the ABEM and the BENCH mechanism map unit prices to quantities. The bid of marginal valuation, $b_{a}^{m a r}$, is therefore given by the inverse of $V_{a}^{\prime}$ :

$$
b_{a}^{\text {mar }}(\rho)=V_{a}^{\prime-1}(\rho)=\frac{1}{2 \delta_{a}}\left(\rho-v_{a}\right)
$$

In order to construct a bid that maximises his surplus, $a$ can submit a bid that deviates from $b_{a}^{m a r}$ - in particular, $a$ could deviate from both his $v_{a}$ and $\delta_{a}$ values. For simplicity, we fix $\delta_{a}$ and restrict $a$ to adapt only the parameter $v_{a}$. In [2], this restriction of the function parametrisation in 
a market mechanism is called "c-parametrisation" (because they used " $C$ " as a parameter name where we use " $v_{a}$ ") and previous literature in which this restriction was also used is described. In a bid $b_{a, r}$, we denote the adapted $v_{a}$ as $v_{a}^{*} . b_{a, r}$ is given by:

$$
b_{a, r}(\rho)=\frac{1}{2 \delta_{a}}\left(\rho-v_{a}^{*}\right)
$$

In the BENCH mechanism, $a$ has, next to $v_{a}^{*}$, also to choose $\rho_{a}^{B}$, his constant price bid for balancing power (see Section 4.1.1).

\subsubsection{Market power of $a$ as a flexible supplier}

Market power is defined as "the ability to alter profitably prices away from competitive levels" [20]. Nicolaisen et al. (2001) [24] distinguish structural market power (which exists in the case where all traders reveal their marginal costs) from strategic market power (which exists in the case where traders misrepresent marginal costs in their bids). We measure $a$ 's realised strategic market power by calculating the Lerner index $\in[0,1]$, defined by dividing per-unit profits by unit price. As the index is defined for a monopolist, we multiply it by $a$ 's market share to compute the Lerner index for an oligopoly [31]:

$$
\operatorname{lerner}\left(q_{a}^{A}, q_{a}^{B}\right)=\frac{\rho_{a}\left(q_{a}^{A}, q_{a}^{B}\right)-\operatorname{costs}_{a}^{a v g}\left(q_{a}^{A}, q_{a}^{B}\right)}{\rho_{a}\left(q_{a}^{A}, q_{a}^{B}\right)} s_{a}\left(q_{a}^{A}, q_{a}^{B}\right)
$$

where $\rho_{a}$ denotes the average unit price which $a$ earns when selling the quantities $q_{a}^{A}$ and $q_{a}^{B}$, costs $a_{a}^{a v g}$ is the average production costs per unit and $s_{a}$ is $a$ 's market share. In our case:

$$
\begin{aligned}
\rho_{a}\left(q_{a}^{A}, q_{a}^{B}\right) & =\frac{q_{a}^{A} \rho_{a}^{A}+q_{a}^{B} \rho_{a}^{B}}{q_{a}^{A}+q_{a}^{B}} \\
\operatorname{costs}_{a}^{a v g}\left(q_{a}^{A}, q_{a}^{B}\right) & =\frac{\operatorname{costs}_{a}^{f u l l}\left(q_{a}^{A}+q_{a}^{B}\right)}{q_{a}^{A}+q_{a}^{B}} \\
s_{a}\left(q_{a}^{A}, q_{a}^{B}\right) & =\frac{q_{a}^{A}+q_{a}^{B}}{q_{a}^{A}+q_{-a}^{A}+q_{-a}^{B}+q_{a}^{B}}
\end{aligned}
$$

where $\operatorname{costs}_{a}^{\text {full }}$ denotes the full production cost function of $a$ and $q_{-a}^{A}$ denotes the amount which all suppliers but $a$ sold in market $A$ (when $a$ is a supplier) or the amount which all consumers bought (when $a$ is a consumer) in market $A . q_{-a}^{B}$ denotes the amount which all suppliers but $a$ sold in market $B$.

Note that this way of modelling market power works well for a supplier, but is not defined for the perspective of a flexible consumer who also acts as a supplier in market $B$.

\subsection{Experiment setup}

\subsubsection{Oligopolistic market scenario}

First, we define an oligopolistic market scenario, which could for instance resemble the situation in a microgrid. Both microgrids and wholesale markets resemble oligopolistic markets, because they are dominated by a small number of players.

Supply side in market $A$ - In [32], several generators and a generic buyer profile are modelled for a simulation of 24 hours. From this study, we model an average generator $g$ and the sum of aggregated demand. Our chosen settings correspond to hour 8am in [32]. We chose that hour as it is similar to most other hours and not an outlier. As was noted earlier, our experiments perform a one-shot auction. Note also that, because we use settings from a wholesale market study, the prices in our model are in $\$ / \mathrm{MWh}$. However, the general findings of this model can also hold for markets which trade $\mathrm{kWh}$, as we only use settings to model relative quantities and slopes of cost functions. 
The average generator $g$ has a maximal production of $q_{g}^{U}=300$ units, a minimum unit cost $v_{g}=18.8$ and $\delta_{g}$, the slope parameter of $g$ 's marginal cost function, is given by $\delta_{g}=0.008$. The model in [32] includes five generators. Thus, to arrive at the average slope of $S^{A}$, we multiply the slope of $g$ 's marginal costs by five: $\beta^{A}=\frac{5}{2 \delta_{q}}$. When we model $a$ as a supplier, then $S_{-a}^{A}$, the aggregated function without $a$, has the slope $\beta^{A}=\frac{4}{2 \delta_{g}}$. Finally, we assume that the minimal unit price of $S_{-a}^{A}$ is $10 \%$ higher than $g$ 's minimal unit costs: $\rho_{\text {min }}^{A}=1.1 v_{g}$.

Demand side in market $A$ - The sum of the demand of all buyers in [32] is 900 , or $3 q_{g}^{U}$. We set $D_{\max }^{A}=3 q_{g}^{U}\left(1-r_{m}\right)$. All studies in [18] measured the price elasticity of demand, which denotes the percentage change in quantity demanded in response to a one percent change in price. [18] distinguishes between "long-run" and "short-run" demand. At the time when he considers the price, a consumer with long-term demand has more time until the time of consumption than a consumer with short-term demand. Thus, having short-term demand allows for less substitution of demanded power with any alternative (e.g. shifting demand to a later time), similar to the situation in a balancing market. The survey reports price elasticities between 0.7 and 2.1 for "long-run" scenarios (which we use for market $A$ ) and between 0.03 and 0.5 in "short-run" scenarios (which we use for market $B$ ). We take $\alpha^{A}=1.0$ and $\alpha^{B}=0.2$.

Coupling of market $B$ - Two parameters of market $B$ are determined by the outcome of market $A$ : the minimum price $\rho_{\min }^{B}$ and the maximal reserve capacity $D_{\max }^{B} . \rho_{\min }^{B}$ is determined as the price at which demand and supply in market $A$ (without $a$ taken into account) intersect $\left(D_{-a}^{A}\left(\rho_{\min }^{B}\right)=S_{-a}^{A}\left(\rho_{\min }^{B}\right)\right)$. We assume that $D_{\max }^{B}$ is related to demand in market $A$ via a ratio $r_{m}$, such that $D_{\max }^{B}=\frac{r_{m} q_{C}^{A}}{1-r_{m}}$, where $q_{C}^{A}$ denotes the sum of all binding commitments of consumers in market $A$ (without $a$ taken into account, if he is a consumer).

Reserve capacity - The $S O$ needs to allocate sufficient reserve capacity from all market participants in market $A$, such that $q^{R} \geq D_{\max }^{B}$. For this, he might approximate $r_{m}$ from experience (refer to Section 1). We assume he is successful in this. For the purposes of this decision-theoretic model, we need to decide which level of reserve capacity agent $a$ bids on ${ }^{1}$, i.e. which $r$ is set in his bid $b_{a, r}$. For the simplicity of our setup, we assume that the $S O$ can approximate $r_{m}$ perfectly and requires $a$ to use $r_{m}$ in his bid $b_{a, r}$ with $r=r_{m}$. This modeling choice assumes that $r$ is technically feasible with the generation or consumption devices that $a$ has. We will use two valued for $r, 0.1$ and 0.3 (see Section 4.2.4). While the smaller value of $r=0.1$ should be feasible with almost all devices, the higher value of $r=0.3$ is mainly possible with devices that have little costs of switching between states, e.g. batteries. Of course, a more detailed model would assume that bidders have individual preferences which values for $r$ they prefer, e.g. based on their devices or previous history. In such a market clearing mechanism, bidders can submit several bids with different values for $r$. We discuss this possibility in Section 2.3 and also propose it for future work (see Section 5).

\subsubsection{Competitive market scenario}

We also design a second scenario (using the oligopolistic scenario as a starting point), in which we model two trends that are considered very important for smart grids. First, we make the scenario more competitive: we increase both the number of suppliers and demand responsiveness tenfold (which affects $\alpha^{A}$ and $\beta^{A}$ ). Second, we add demand (e.g. to model increasing market penetration of electric vehicles and heat pumps) by doubling the overall demand for electricity (which affects $D_{\max }^{A}$ ). Table 2 lists all default parameter values for the two scenarios. Note that the parameters for market $B$ depend on the parameters of market $A$.

\subsubsection{Settings for bidding agent $a$}

Participation in an ABEM market is simulated for two types of bidders, a flexible supplier and a flexible consumer. They are flexible to supply more or consume less, respectively, on short notice

\footnotetext{
${ }^{1}$ All inflexible actors use $r=0$ in their bids. As all market participants other than $a$ are modelled by unconstrained functions, we do not need to decide which values of $r$ the flexible participants bid.
} 
Table 2: Default settings for parameters in the oligopolistic and competitive scenario.

\begin{tabular}{ccc}
\hline Name & oligopolistic scenario & competitive scenario \\
\hline$D_{\max }^{A}$ & $3 q_{g}^{U}\left(1-r_{m}\right)$ & $6 q_{g}^{U}\left(1-r_{m}\right)$ \\
$\alpha^{A}$ & 1.0 & 10.0 \\
$\rho_{\min }^{A}$ & $1.1 v_{g}$ & $1.1 v_{g}$ \\
$\beta^{A}$ & $\frac{5}{2 \delta_{g}}$ & $\frac{50}{2 \delta_{g}}$ \\
$D_{\max }^{B}$ & $\frac{r_{m} q^{A}}{1-r_{m}}$ & $\frac{r_{m} q^{A}}{1-r_{m}}$ \\
$\alpha^{B}$ & $\frac{\alpha^{A}}{5}$ & $\frac{\alpha^{A}}{5}$ \\
$\rho_{\min }^{B}$ & $\rho_{-a}^{A}$ & $\rho_{-a}^{A}$ \\
$\beta^{B}$ & $\beta^{A}$ & $\beta^{A}$ \\
$r_{m}$ & 0.1 or 0.3 & 0.1 or 0.3 \\
\hline
\end{tabular}

(this ancillary service is usually referred to as "upward regulation").

In order to model $a$ as a flexible supplier, we parametrise $a$ as an average generator, according to [32] (see Section 4.2.1). We set $v_{a}=v_{g}, \delta_{a}=\delta_{g}$ and $q_{a}^{U}=q_{g}^{U}$.

We model $a$ as a flexible consumer in the following way: His maximum capacity is $q_{a}^{U}=q_{g}^{U}$, same as for our average generator $g$ (see Section 4.2.1). For the slope of the valuation function of a flexible consumer, literature does not provide us with helpful pointers. For this work, we choose $\delta_{a}=-0.008$, mirroring $\delta_{g}$, the slope of the cost function of $g$. Finally, we aim at modeling $a$ 's utility function $V_{a}$ such that $a$ 's valuation is close to the market valuation and set $v_{a}=\rho_{-a}^{A B} * 1.1$. $\rho_{-a}^{A B}$ is the average price over markets $A$ and $B$, under given parameter settings, if $a$ is not present. The multiplication by 1.1 roughly compensates for the slope $\delta_{a}$.

\subsubsection{Method}

In the experiments, we evaluate both the oligopolistic and the competitive market scenario using a Monte-Carlo simulation. We now describe the generation of specific parameter settings, the method of sampling traces in them, and which steps bidding agent $a$ follows to find optimal bid parameters.

Parameter settings - We create several relevant settings in both market scenarios by varying the value of one parameter a a time, where the other parameters remain at the default setting from Table 2. In both scenarios, $\phi \in[0,3]$ ( $\phi$ is the uncertainty scaling parameter and will be explained below), $\rho_{\min }^{A} \in\left[\frac{2}{3} v_{g}, \frac{3}{2} v_{g}\right]$ and $D_{\max }^{A} \in\left[\frac{2}{3} D_{\max }^{A \text {,base }}, \frac{3}{2} D_{\max }^{A, \text { base }}\right]$, where $D_{\max }^{A \text {,base }}$ denotes the default setting for $D_{\max }^{A}$ from Table 2. Note that $\rho_{\min }^{B}$ and $D_{\max }^{B}$ are formulated in relation to $\rho_{\min }^{A}$ and $D_{\max }^{A}$, respectively. Finally, we run simulations with $r_{m}=0.1$, which is a reserve level observed often in current markets, as well as $r_{m}=0.3$, a setting that is not unrealistic in the market scenarios we can expect in the upcoming 10 years, at least for the actors that can offer significant reserve power (for example if they operate batteries or gas power plants).

Sampling - We sample the outcomes for each setting 100 times. Each sample contains a new pair of the noise parameters $k^{A}$ and $k^{B}$, which influence the position of the residual functions that $a$ faces with respect to quantity (see Section 3.4). Each pair is generated by the Mersenne twister pseudo-random number generation algorithm. We assume that the two probability distribution functions $\mathrm{prob}^{A}$ and $\mathrm{prob}^{B}$ are independent from one another. Also, we assume they model normal distributions and we thus have to make two choices: how to set means and standard deviations for the distribution functions of both $k^{A}$ and $k^{B}$. First, we set both means to 1 , which is the value for which there is no noise, compare Equation 22. Second, we define the standard deviations $s^{A}$ and $s^{B}$ such that the position of the residual function for $a$ in the given market is changed by a certain amount (the amount is specific to the market setting). We explain this definition using $k^{B}$ in market $B$ as an example. At a noise value of $k^{B}=1$, no noise is present. Residual demand is not willing to buy from $a$ above the price $\rho_{-a}^{B}\left(D_{\text {res }}^{B}\left(\rho_{-a}^{B}, 1\right)=0\right)$. We define $s^{B}$ such that $D_{\text {res }}^{B}\left(\rho_{\text {min }}^{B}, 1-3 s^{B}\right)=0$. Thus, a value of $k^{B}=1-3 s^{B}$ repositions $D_{\text {res }}^{B}$ downwards along 
the quantity axis such that residual demand is not willing to buy from $a$ above the price $\rho_{\min }^{B}$. Finally, in order to model varying degrees of noise, we create additional parametrised settings, where we vary $s^{A}$ and $s^{B}$. To create these settings, we multiply both $s^{A}$ and $s^{B}$ with a scaling parameter $\phi \in[0,3]$ (in default settings, $\phi=1$ ).

Finding optimal bid parameters - in Section 4.1.2, we describe that bidding agent $a$ has to optimise one (in the ABEM mechanism) or two (in the BENCH mechanism) bid parameters. He does this in two steps. First, $a$ performs a brute-force search on parameter settings for his bid(s). $a$ evaluates 100 evenly-spaced values for $v_{a}^{*}$ (in his bid to market $A$ ) in the range $\left[v_{a}, \rho_{\max }^{A}\right]$ when $a$ is a supplier or $\left[\rho_{m i n}^{A}, v_{a}\right]$ when $a$ is a consumer. Furthermore, when participating in the BENCH market, $a$ evaluates, for each of the 100 values he evaluated for $v_{a}^{*}, 100$ evenly-spaced values for $\rho_{a}^{B}$ (his bid to market $B$ ) in the range $\left[p_{\min }^{A}, \rho_{\max }^{B}\right]$. Here lies the main difference in the time it takes to compute a bid with optimal expected value. In our experiments, optimising a bid in the ABEM mechanism took around a minute, while optimising bids to the BENCH mechanism took up to around one hour on a standard desktop PC.

The second step of the bid optimisation is to refine the best solution from the brute-force search. Starting with the most promising bid(s) found so far (with respect to his expected surplus), $a$ applies a downhill simplex algorithm [23] to maximise the expected surplus further.

During the evaluation of each value setting, $a$ sets $k_{\min }^{A}=1-3 s^{A}, k_{\max }^{A}=1+3 s^{A}, k_{\min }^{B}=$ $1-3 s^{B}$ and $k_{\max }^{B}=1+3 s^{B}$ (refer to Equation 29).

\subsection{Results and Discussion}

\subsubsection{Agent $a$ as a flexible supplier}

We begin with confirming that, for several general economic properties, the market model behaves as expected in reality. First, $a$ has positive surplus in both mechanisms and across all settings. $a$ 's surplus also correlates with settings like one would expect. It is positively correlated to changes in $\delta_{a}, D_{\max }^{A}$ and $\rho_{\min }^{A}$ and negatively correlated to $r_{m}, \alpha^{A}$ and $\beta^{A}$. Second, $a$ 's presence increases competition as he can offer electricity below market price. We simulated the markets without $a$ (thus decreasing the number of suppliers by one). As should be expected, the aggregated unit price (the sum of all sold units in both markets divided by the sum of money paid by consumers) is significantly higher in these settings than it is with $a$ 's presence. Finally, in comparison to the oligopolistic scenario, the competitive scenario has a lower aggregated market price, as well as lower market power and surplus for $a$.

We now turn to two important observations, concerning notable differences or similarities in outcomes when $a$ takes part in either the ABEM or the BENCH mechanism:

Observation 1: Agent a reaches comparable surplus in both mechanisms across a wide range of market conditions, but shows different bidding behaviour. The outcomes for $a$ are different, between both mechanisms, in terms of quantities $a$ supplies and prices $a$ is paid. The differences are most prominent in market $B$ and we now note two notable ones. First, the results for $a$ in market $B$ vary mostly in price in ABEM, while in BENCH, they vary mostly in quantity (see Figure 5 for two examples). Second, $a$ in the BENCH mechanism does not sell any $q_{a}^{B}$ in settings with high uncertainty $(\phi>1.5)$, because he charges a price that is too high for the market.

However, $a$ 's surplus does not differ significantly ${ }^{2}$ across all settings when we let $a$ take part in the ABEM or BENCH mechanism. The only exceptions are in the oligopolistic scenario when the setting has high values for $D_{\max }^{A}$ (where $a$ has higher surplus in ABEM) or in both scenarios when the setting has high values for $\rho_{\min }^{A}$ (where $a$ has higher surplus in $\mathrm{BENCH}$ ).

Observation 2: The ABEM mechanism substantially reduces market prices and market power in exploitable settings. In the default settings, market power measurements for

\footnotetext{
${ }^{2}$ We performed Student's T-Tests and tested for $p \leq 0.01$.
} 


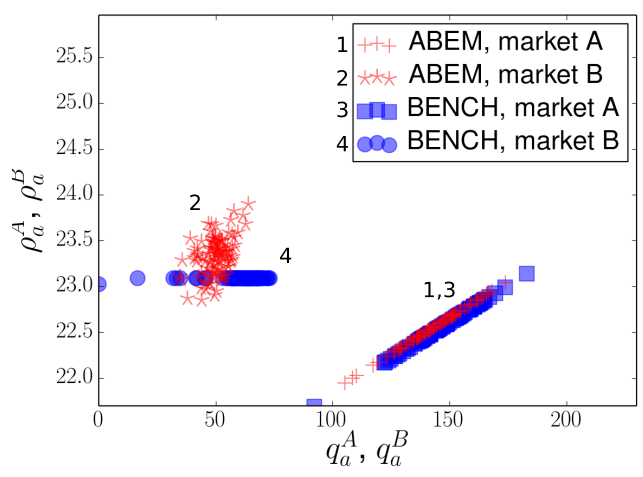

(a) oligopolistic scenario, $r_{m}=0.3$

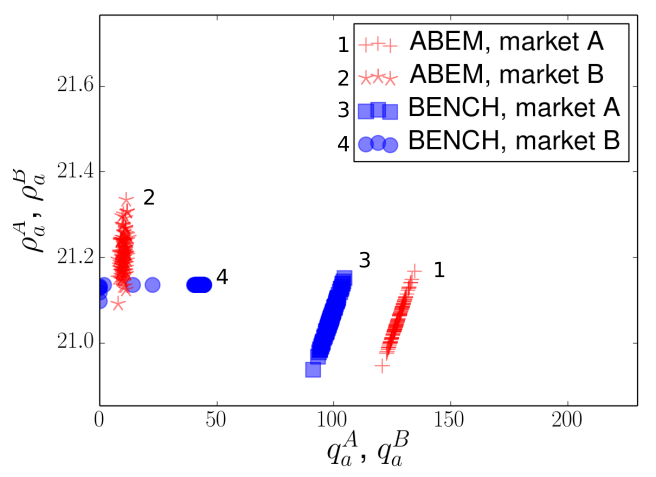

(b) competitive scenario, $r_{m}=0.3$

Figure 5: Sampled outcomes for $a$ in default settings. The outcomes of different runs have been labelled 1 through 4 for convenience.

agent $a$ in the ABEM and BENCH mechanism show no significant differences. The biggest opportunities for $a$ to exercise market power exist in settings with larger values for $\rho_{m i n}^{A}$, because then the difference between offer prices of $S_{-a}$ and $a$ 's costs is high and $a$ can thus increase his surplus. The settings in which $\rho_{m i n}^{A} \geq 24$ show by far the highest aggregated market prices, as well as market power and surplus for $a$. In these settings, $a$ exploits this opportunity less when he uses the ABEM mechanism (see Figure 6 for an example). The differences in $a$ 's market power between the ABEM and the BENCH mechanism in these settings are significant, with the exception of the oligopolistic scenario where $r_{m}=0.1$. This observation also aligns with some differences in surplus which we reported in observation 1 (in settings with high values for $\rho_{\min }^{A}$ ).

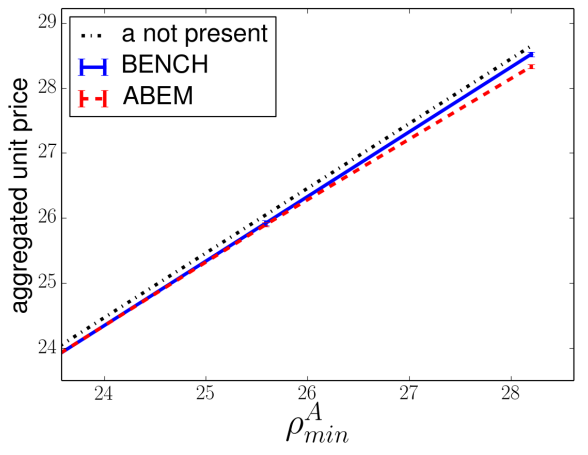

(a) Aggregated unit prices across both markets.

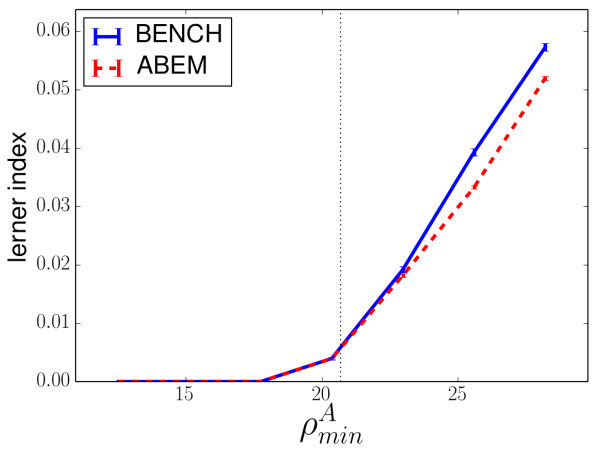

(b) Market power of $a$.

Figure 6: Effects of increasing prices of $a$ 's competition in the competitive scenario, $r_{m}=0.3$. Results shown with ${ }_{-}^{+} 1$ standard deviation.

Specifically, $a$ in ABEM is lowering the price $\rho_{a}^{A}$ on market $A$, and as a result the aggregated market prices are lower than in the BENCH mechanism. Note that the most quantity is sold on market $A$ and thus lowering $\rho_{a}^{A}$ has a strong effect. See Figure 6a for the most substantial case, where the presence of $a$ when using the ABEM mechanism has an impact on aggregated market prices which is up to 2.7 times larger as when $a$ takes part in the BENCH mechanism. The results also show a clear reduction in market power. In the default settings $\left(\rho_{\min }^{A}=20.68\right), a$ has the same market power in both mechanisms. However, in settings with $\rho_{\min }^{A} \geq 24, a$ gains substantially less market power with respect to the default setting when taking part in the ABEM mechanism and 
therefore has less market power than when taking part in the BENCH mechanism. See Figure 6b for the most substantial case, where $a$ has up to $11 \%$ less market power in the ABEM mechanism.

Discussion: First, the mechanisms ABEM and BENCH prescribe different bid formats for market $B$. This leads to different bidding behaviour by $a$ in both markets (see observation 1). In market $B$, agent $a$ in BENCH bids a constant price $\rho_{a}^{B}$ and thus the results differ only along the quantity axis (for $q_{a}^{B}$ ). Agent $a$ in ABEM, on the other hand, bids a supply function to market $B$, and thus results for both $q_{a}^{B}$ and $\rho_{a}^{B}$ differ (depending on $\delta_{a}$, the slope of $a$ 's bid).

Most important, however, is the confirmation that $a$ reaches the same level of surplus in ABEM and in BENCH. This shows that using ABEM is economically as rewarding as our benchmark mechanism BENCH. The observation that $a$ in the BENCH mechanism does not sell any $q_{a}^{B}$ in settings with high uncertainty is more prevalent when $a$ is a flexible consumer, so we will discuss this behaviour in Section 4.3.2.

We now turn to observation 2 and discuss bidding behaviour under exploitable market settings (here modelled by large values for $\rho_{m i n}^{A}$ ). In most settings we simulated, multiple near-optimal combinations of quantities and prices exist. Though bids in the ABEM mechanism are less flexible than in the BENCH mechanism (because only one bid function can be submitted), $a$ is likely to find a bid $b_{a, r}$ that realises one of them, as is evident in the good performance across all settings (see observation 1). However, the market settings in question (with $\rho_{\min }^{A} \geq 24$ ) are so favourable for $a$ that he can sell all capacity on both markets $\left(q_{a}^{A}=q_{a}^{U}\left(1-r_{m}\right)\right.$ and $\left.q_{a}^{B}=q_{a}^{R}\right)$. This means that there exists only one pair of optimal quantities (because bid functions are monotonically increasing) and the optimisation problem is reduced to finding the optimal prices for this pair of quantities.

However, in the ABEM mechanism the following restriction is in place: Let the quantity $q_{a}^{A}$ be fixed. Then, the distance between bids $b_{a, r}$ and $b_{a}^{B}$ with regard to the price is fixed as well (because the minimum price of $b_{a}^{B}$ is $v_{a}^{*}+2 \delta_{a} q_{a}^{A}$ ). Thus, in ABEM it becomes highly unlikely that $a$ can bid optimal prices in both markets in this situation. We conclude that in conditions with excessive market power for $a$, the ABEM mechanism restricts $a$ from realising the full potential market power. In effect, $a$ in the ABEM mechanism lowers bid $b_{a, r}$ substantially, in order to not overprice on market $B$. It makes the ABEM mechanism appealing to market makers, as it protects vulnerable consumers from unnecessarily high prices.

\subsubsection{Agent $a$ as a flexible consumer}

As we do in Section 4.3.1, we begin by validating the market model for several important properties: $a$ 's overall surplus is positive in both mechanisms across all settings and, in comparison to the oligopolistic scenario, the competitive scenario has lower market prices and a lower surplus for a. We now discuss two major observations, where observation 1 is similar to observation 1 in Section 4.3.1.

Observation 1: Agent a reaches comparable surplus in both mechanisms across a wide range of market conditions, but shows different bidding behaviour. The outcomes for $a$ show distinct patterns between both mechanisms, most prominently in market $B$. If $a$ sells electricity in both markets, this resembles outcomes we showed in Figure 5 and described in Section 4.3.1. However, in many settings, $a$ in the BENCH mechanism sells no $q_{a}^{B}$ at all (see observation 2 for more details on this).

Despite such differences in market outcomes, $a$ 's surplus does not differ significantly ${ }^{3}$ across all settings when we let $a$ take part in the ABEM or BENCH mechanism. This observation is present in all our parametrised market settings, with the only exception for low values of $\rho_{\min }^{A}$ (where $a$ has higher surplus in the BENCH mechanism).

\footnotetext{
${ }^{3}$ We performed Student's T-Tests and tested for $p \leq 0.01$.
} 


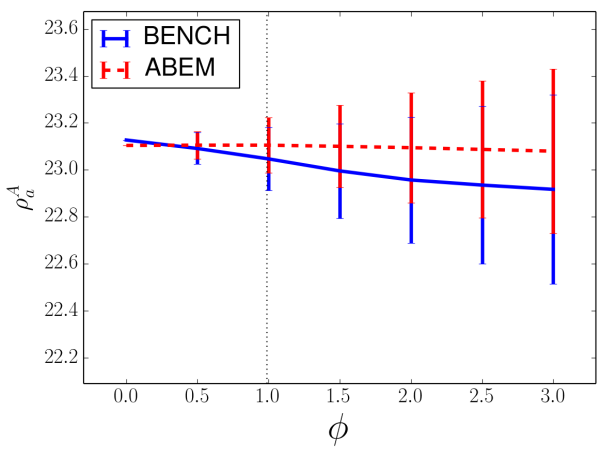

(a) Effects on $\rho_{a}^{A}$

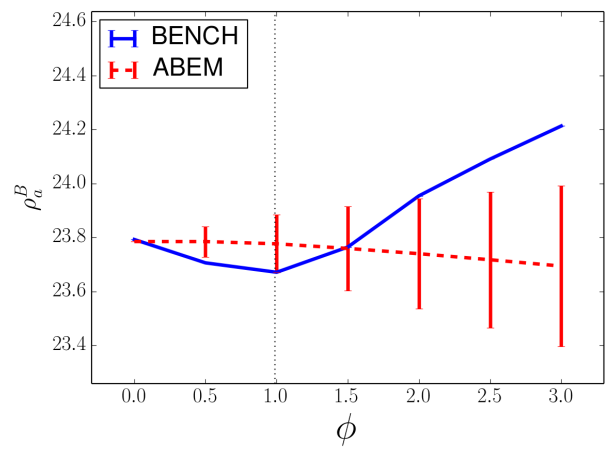

(b) Effects on $\rho_{a}^{B}$

Figure 7: Effects of increasing uncertainty on prices for $a$ (oligopolistic scenario, $r_{m}=0.3$ ). The dotted line shows the default setting $\phi=1$.

Observation 2: Agent $a$ offers and sells reserve capacity at affordable prices in the ABEM mechanism, but not in the BENCH mechanism. In the ABEM mechanism, $a$ consistently sells balancing power across most market settings, at prices which inflexible actors are willing to pay. $a$ in BENCH, however, will in many settings bid a price $\rho_{a}^{B}$ which is too high in the given market setting. As a consequence, he sells, when compared to the ABEM mechanism, very little $q_{a}^{B}$ or even none at all. In fact, $a$ in BENCH only sells $q_{a}^{B}$ in the oligopolistic scenario, when $r_{m}=0.3$, neither $\rho_{\min }^{A}$ nor $D_{\max }^{A}$ are low and $\phi$ is not high. Figures 7 and 8 illustrate what is happening when the uncertainty parameter $\phi$ ( $a$ 's uncertainty about market outcomes increases with $\phi$, refer to Section 4.2.4) is varied. $a$ in the BENCH mechanism increases the price $\rho_{a}^{B}$ and sells less $q_{a}^{B}$ when $\phi>1$. At the same time, he decreases the price on market $A$ and thus buys $q_{a}^{A}$ cheaper than $a$ in ABEM.

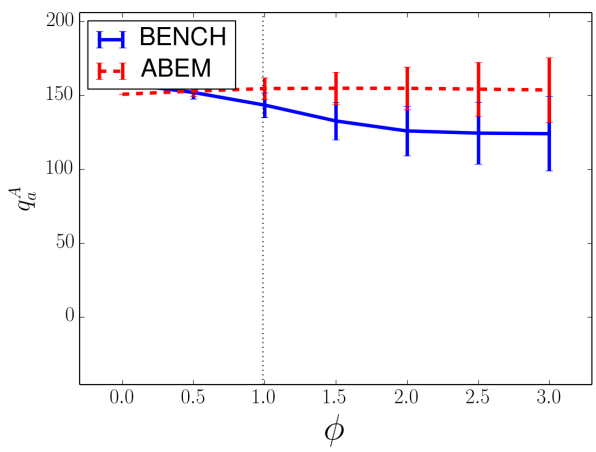

(a) Effects on $q_{a}^{A}$

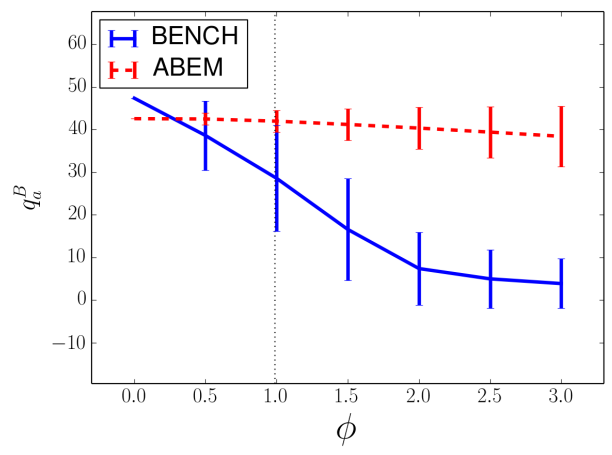

(b) Effects on $q_{a}^{B}$

Figure 8: Effects of increasing uncertainty on quantities for $a$ (oligopolistic scenario, $r_{m}=0.3$ ). The dotted line shows the default setting $\phi=1$.

Discussion: The fact that agent $a$ reaches comparable surplus in both mechanisms (see observation 1) confirms observation 1 in our first experiment (see Section 4.3.1) where $a$ is a flexible supplier and the same discussion applies here. The only exception is given in settings with low values for $\rho_{\min }^{A}$. We will discuss this exception in our discussion of observation 2 .

Observation 2 describes that $a$ in BENCH is only in a few settings willing to offer his reserve capacity at prices which are acceptable to inflexible actors in market $B$ (and thus does not sell $q_{a}^{B}$ 
in other settings). This observation makes the ABEM mechanism attractive to market operators.

Agent $a$ in BENCH does offer and sell $q_{a}^{B}$ at affordable prices in the default settings of the oligopolistic scenario. In the remainder of this section, we will discuss the three settings noted in observation 2 (low values for $\rho_{\min }^{A}$, low values for $D_{\max }^{A}$, high values for $\phi$ ), which lead $a$ in BENCH to overprice $q_{a}^{B}$ in the oligopolistic scenario and also explain why he does not sell $q_{a}^{B}$ at all in the competitive scenario. Keep in mind, however, that settings with low values for $\rho_{\min }^{A}$ are the only ones where surplus for $a$ differs significantly between the ABEM and BENCH mechanisms (see observation 1). In all other settings, the difference from $a$ 's point of view is merely one of bidding strategy choices, which $a$ makes, not of financial outcomes for $a$. This is significant, as we should require that bidders are able to make the same amount of surplus as in comparable mechanisms.

In general, $a$ in BENCH behaves as observation 2 describes, in order to avoid costs of lost opportunity. In particular, there are two reasons these costs exists. First, if $a$ is uncertain about market outcomes, the financial risk of buying electricity in market $A$ with the goal of offering it as reserve capacity in market $B$ becomes too high for him when he bids a constant price $\rho_{a}^{B}$ (as prescribed by the BENCH mechanism). Second, in some settings, $a$ in the BENCH mechanism can buy electricity at a very low price $\rho_{a}^{A}$ in market $A$ and the price difference between $\rho_{a}^{A}$ and possible prices $\rho_{a}^{B}$ in market $B$ is not attractive enough. We now begin by discussing settings where the latter reason for not selling $\rho_{a}^{B}$ holds.

In settings with low values for $\rho_{\text {min }}^{A}$, supply prices are low in market $A$ and thus an opportunity exists for buyers to raise their surplus considerably. $a$ buys $q_{a}^{A}$ far under his reserve price $v_{a}$. In the BENCH mechanism, $a$ does not need to sell any $q_{a}^{B}$ (he can overprice, as observation 2 describes), while $a$ in the ABEM mechanism is required to sell $q_{a}^{B}$ at a price related to the price he paid for $q_{a}^{A}$. Settings with low values for $D_{\max }^{A}$ are similar - $q_{a}^{A}$ can be bought cheaply in market $A$ (in this case, because there is little competition from other buyers) and $a$ in BENCH prefers not to offer it in market $B$ at the prices he could achieve there. Finally, in the competitive scenario, the number of suppliers is increased tenfold, which decreases prices in both markets. As a consequence, buying on market $A$ becomes more attractive and selling on market $B$ becomes less attractive, which leads to $a$ in BENCH to not sell $q_{a}^{B}$.

We now turn to settings with high values for $\phi$, in other words, with high uncertainty $a$ has about market outcomes. In the BENCH mechanism, both the bid to market $B\left(\rho_{a}^{B}\right)$ and the residual demand function for balancing power $\left(D_{\text {res }}^{B}\right)$ react only very little to changes in price: The former is a constant price, and the latter has a low slope. The intersection of both varies strongly along the quantity axis. Figure $8 \mathrm{~b}$ ) illustrates this. Consider for example the default setting, where $\phi=1$ (a base level of noise exists) and $a$ still sells $q_{a}^{B}$ in BENCH. This variation of possible quantities for $a$ means that, in the BENCH mechanism, $a$ is facing a higher risk than in the ABEM mechanism, if he follows the strategy of increasing his bid to market $A$ (i.e. to pay a higher price for $q_{a}^{A}$ ), in order to be able to offer reserve capacity $q_{a}^{R}$ and sell $q_{a}^{B}$. The negative outcome $a$ risks in this case is that he might sell too little or no $q_{a}^{B}$ due to noise in market $B$. This would strongly lower $a$ 's overall surplus. Therefore, $a$ lowers his bid $b_{a, r}$ to market $A$, in order to optimise surplus there, and overprices his bid price $\rho_{a}^{B}$ in market $B$, in order not having to sell any $q_{a}^{B}$.

This is not the case in the ABEM mechanism. Here, $a$ submits a positively-sloped bid function to market $B$, which reduces variation along the quantity axis. This result relates to the original paper about supply function equilibria by Klemperer and Meyer (1989) [15], where the authors note that, when faced with exogenous uncertainty about residual demand, "a supply function provides valuable flexibility, because it can be chosen to coincide with the set of optimal pricequantity pairs". By using supply functions in market $B$ rather than a constant price (a Bertrand model), the ABEM mechanism is allowing for a high degree of detail in the expression of economic value by bidders. 


\section{Conclusions and related work}

Because peaks in both electricity generation and consumption are expected to increase in future energy settings, the "smart grid" concept aims to maintain high levels of efficiency in the energy system by establishing distributed intelligence. Dynamic decisions will be made by many intelligent agents (with unknown computational capabilities) in autonomous fashion, on behalf of households and devices. Market mechanisms are a promising approach for large-scale coordination problems about energy supply and demand, but in order to reach satisfactory efficiency levels, it is crucial to involve planning-ahead by letting capable participants commit early, in either a binding or an optional manner. It has become common practice in several wholesale electricity markets around the world to conduct planning-ahead in both of these ways. However, existing proposals for electricity markets in smart grids, with high number of participants, either do not involve planning-ahead sufficiently or require a high level of sophistication and considerable computing power from participants.

In this paper, we present and evaluate a novel market mechanism called ABEM ("Aheadand Balancing Electricity Market"). ABEM is an effective market mechanism that incorporates planning-ahead in the way modern wholesale markets do, but is also usable for bidders with non-sophisticated computational capabilities, i.e. in smart grids. We provide three main contributions ${ }^{4}$, as follows.

1. We introduce the ABEM mechanism (Section 2). The ABEM mechanism has two unique features: Bids for binding commitments as well as for reserve capacity are combined into one bid, and this bid specifies a quantitative relationship between binding commitments and reserve capacity. ABEM provides several main advantages by design: First, the bid optimisation problem for bidding agents is reduced in complexity from a two- to a onedimensional problem, which significantly reduces the time necessary to compute well-working bids. Second, agents can bid price functions to both markets, potentially their true costs or valuation, which is usually problematic in other approaches. Third, the mechanism provides a guarantee for flexible consumers that offering reserve capacity is profitable (if they submit their marginal valuation as bid).

2. We provide a parametrised market model (Section 3) of a two-settlement market with integrated trade of reserve capacity and simultaneous bidding (which can be used to represent ABEM, among others). A strategic bidding agent can use this market model for his bid optimisation problem, using a decision-theoretic approach. The market model is fully parametrised, most importantly with respect to the uncertainty the agent has about market outcomes. We also formulate the bid optimisation problem an agent needs to solve when using this model.

3. We evaluate ABEM experimentally, with respect to a benchmark mechanism (Section 4). This approach was chosen because a theoretical analysis of possible outcomes is not feasible for such complex settings as the one described above. We simulate both an oligopolistic and a competitive market scenario using parametrised Monte Carlo simulations. Participation in an ABEM market is simulated for two types of bidders, a flexible supplier and a flexible consumer. The computational simulations show that, from the bidding agent's perspective, the same levels of overall economic surplus (on average across all tested settings) can be reached in both ABEM and the benchmark mechanism. The simulations also confirm that the bidding agent's bids are much more efficiently computable if he takes part in ABEM. Furthermore, from the system operator perspective, we show experimentally two advantages of ABEM over the benchmark mechanism. First, bidding agents make less use of strategic opportunities to exploit market settings, which keeps overall prices low. Second, reserve

\footnotetext{
${ }^{4}$ We note that some preliminary concepts and results of what today comprises the ABEM mechanism have been presented across three conference papers $[9,10,11]$ in recent years. This paper provides deeper background on related work and economical foundations. It describes the mechanism in more detail and with coherent nomenclature. Finally, it also presents all experimental and theoretical results in a coherent fashion.
} 
capacity for balancing is made available at affordable prices, whereas in the benchmark mechanism it would often be overpriced, in order to avoid costs of lost opportunity.

\subsection{Related work}

Several state-of-the-art mechanisms in wholesale electricity markets implement all or a subset of the features mentioned in Section 2.1. For instance, several two-settlement markets are currently implemented, e.g. in New England [12] and The Netherlands [33]. While the U.S. Midcontinent energy market [22] is the only mechanism known to us which combines all of the features mentioned in Section 2.1, several two-settlement procedures which also trade reserve capacities or so-called ancillary services differ only in few aspects (for instance they might not require simultaneous bidding). Through several decades of real-world experience in electricity market design, these mechanisms do involve planning to a satisfactory degree, by including binding commitments as well as reserve capacity. However, as they consist of at least two or even three markets ${ }^{5}$, they have several bottlenecks.

Firstly, the computational complexity of creating bids that work well across several markets is very high. This can become problematic in smart grid settings due to the limited computational power and lower predictability (due to intermittent generation), as was discussed earlier. Secondly, from the SO perspective, it is difficult to create the proper economic incentives for bidders. For example, Kamat et al. (2002) [13] report that holding back bids in the ahead market often becomes a profitable strategy to increase prices during balancing. Vandezande et al. (2010) [34] observe that often strong incentives exist to not offer reserve capacity on public markets at all, but rather balance privately owned assets only. Thirdly, the expression of the proper economic valuation of bidders is restricted. As we noted above, the simultaneous submission of bids in the ahead market for binding commitments or for reserve capacity has been identified as good practice to reduce strategic behaviour. However, bidders have to create the bid for reserve capacity without knowing their binding commitment. Although both bids describe the same resource (electricity), the two bids usually contain no explicit relationship to each other. Another restriction of the expression of the proper economic valuation concerns the allowed bid function format. Although using price functions (e.g. piecewise-linear or quadratic) in bids has been shown to increase efficiency of a market $[4,39]$, current wholesale market designs (e.g. the U.S. Midcontinent energy market [22]) mostly require constant prices as bids for reserve capacity in order to deal with the involved complexity. In ABEM, we will address these above bottlenecks.

Besides two-settlement-procedures for wholesale markets, the field of electrical engineering has (often in collaboration with economics) also proposed several market mechanisms with more smart grid-like settings in mind. These mechanisms achieve less complexity and thus better scalability. However, they either lack the ability of planning, do not consider strategic behaviour or have uncertain convergence characteristics. For instance, Kok et al. (2005) [16] propose the PowerMatcher control structure for decentralised energy systems. This system is scalable (i.e. it deals with computational complexity) by the introduction of an hierarchical structure, but does not involve planning-ahead. Bakker et al. (2010) [1] describe a three-step methodology for planning in smart grids, which is also scalable. However, the method assumes a cooperative setting, i.e. it is assumed that bidding agents (e.g. on freezers or heat pumps) are not acting strategically in selfinterest. Virag et al. (2011) [36] also target at an improved mechanism for simultaneous bidding on binding and commitments and reserve capacity. They propose an iterative market design, where in each round the mechanism proposes two market prices and the agents reply with what they would buy at those prices. This is done until convergence. It is assumed that no strategic behaviour by bidders takes place, but even for this case, the convergence of this mechanism is either slow or dependent on initialisation values.

The need for scalable mechanisms (for smart grids) has attracted attention from computer scientists. Several market mechanisms for smart grids have therefore been proposed in computer

\footnotetext{
${ }^{5}$ Some modern wholesale markets add even more components to their mechanisms, e.g. financial transmission rights markets or locational marginal pricing.
} 
science in recent years. For instance, Lamparter et al. (2010) [19] discuss an agent-based market framework which is scalable but lacks features for planning-ahead. Vytelingum et al. (2010) [37] propose a two-settlement procedure. Reserve capacity is not traded explicitly. Instead, unmatched (incremental) offers from the day-ahead market are used for balancing, if needed. This approach thus treats every unmatched bid to the ahead market as reserve capacity. However, this makes the assumption that all bidders can, on short notice and on request, physically change the amount they deliver or consume, which might not hold for all appliances. Furthermore, it is assumed that bidders do not have opportunity costs (e.g. that a consumer will not change his plans if he does not get his desired commitment in the day-ahead market). To include such opportunity costs in his bid is very difficult for a bidder in this mechanism. Other mechanisms have been designed for a specific smart grid-related application only, and are thus not generically applicable as a market mechanism for smart grids. For instance, Vytelingum et al. (2010) [38] look into micro-storage in the domestic households and provide agent-based strategies for planning battery storage profiles for the upcoming day, given a stylised spot market for electricity. Gerding et al. (2011) [6], as well as Stein et al. (2012) [30], address the problem of charging a fleet of electric vehicles, where the former paper proposes a mechanism for plug-in hybrid vehicles and the latter a mechanism for pure electric vehicles. Both papers design a mechanism that is capable of allocating electricity efficiently among vehicles, based on their drivers announced preferences for their next trip.

\subsection{Future work}

For future work, it can be useful to allow bidders to bid on several reserve ratios $r$ at the same time, with several bids to the same market mechanism. This would enable the $S O$ to increase market efficiency by increasing the number of alternative market clearing solutions. The design challenge here is to allow bidding agents some freedom on their choice of values for $r$, but also to give the $S O$ a way to ensure that he will be able to allocate sufficient reserve capacity. The explicit notion of $r$ which ABEM prescribes is a good foundation for solving this problem, as opposed to the static heuristics which are in use today.

\section{References}

[1] V. Bakker, M.G.C. Bosman, A. Molderink, and J.L. Hurink. Demand side load management using a three step optimization methodology. In First IEEE International Conference on Smart Grid Communications, pages 431-436, 2010.

[2] R. Baldick. Electricity market equilibrium models: the effect of parametrization. IEEE Transactions on Power Systems, 17(4):1170-1176, November 2002.

[3] J. Bower and D. Bunn. A model-based comparison of pool and bilateral market mechanisms for electricity trading. Energy Journal, 21(3):1-29, 2000.

[4] M.B. Cain and F.L. Alvarado. Implications of cost and bid format on electricity market studies: linear versus quadratic costs. In Large Engineering Systems Conference on Power Engineering, number July, 2004.

[5] Department Of Energy. Impacts of the Federal Energy Regulatory Commission's Proposal for Standard Market Design. 2003.

[6] Enrico H Gerding, Valentin Robu, Alex Rogers, and Sebastian Stein. Online Mechanism Design for Electric Vehicle Charging. In Proceedings of the 10th International Conference on Autonomous Agents and Multiagent Systems (AAMAS), pages 811-818, 2011.

[7] D. Goldfarb and A. Idnani. A numerically stable dual method for solving strictly convex quadratic programs. Mathematical Programming, 27(1):1-33, September 1983. 
[8] E. Hirst and B. Kirby. Ancillary Services: The Neglected Feature of Bulk-Power Markets. Electricity Journal, 11(3):50-57, 1998.

[9] N. Höning, H. Noot, and H. La Poutré. Integrating power and reserve trade in electricity networks [extended abstract]. In Proceedings of the 10th International Conference on Autonomous Agents and Multiagent Systems (AAMAS), 2011.

[10] Nicolas Höning and Han La Poutré. Flexible Consumers Reserving Electricity and Offering Profitable Downward Regulation. In Third IEEE PES Conference On Innovative Smart Grid Technologies (ISGT), 2012.

[11] Nicolas Höning and Han La Poutré. Reduction of Market Power and Stabilisation of Outcomes in a Novel and Simplified Two-Settlement Electricity Market. In The 2012 IEEE/WIC/ACM International Conference on Intelligent Agent Technology (IAT), Macau, 2012.

[12] ISO New England Inc. 2009 Annual Markets Report. Technical report, ISO New England, 2009.

[13] R. Kamat and S.S. Oren. Rational buyer meets rational seller: Reserves market equilibria under alternative auction designs. Journal of Regulatory Economics, 2002.

[14] Rajnish Kamat and Shmuel S Oren. Two-settlement Systems for Electricity Markets under Network Uncertainty and Market Power. Journal of regulatory economics, 25(1), 2004.

[15] P.D. Klemperer and M.A. Meyer. Supply function equilibria in oligopoly under uncertainty. Econometrica, 57(6):1243-1277, 1989.

[16] J. K. Kok, C. J. Warmer, and I. G. Kamphuis. PowerMatcher: multiagent control in the electricity infrastructure. International Conference on Autonomous Agents and Multiagent Systems, 2005.

[17] V. Krishna. Auction Theory. Academic Press, San Diego, 2002.

[18] R. Lafferty et al. Demand responsiveness in electricity markets. Technical report, Federal Energy Regulatory Commission, 2001.

[19] Steffen Lamparter, Silvio Becher, and J.G. Fischer. An Agent-based Market Platform for Smart Grids. In Proc. of 9th Int. Conf. on Autonomous Agents and Multiagent Systems (AAMAS 2010), pages 1689-1696, Toronto, 2010.

[20] A. Mas-Collel et al. Microeconomics. Oxford University Press, 1995.

[21] Leonardo Meeus. Is the prevailing wholesale market design in Europe and North America comparable? Power Engineering Society General, pages 1-5, 2007.

[22] Midwest ISO. Energy and Operating Reserve. Technical report, 2010.

[23] J.A. Nelder and R. Mead. A simplex method for function minimization. The computer journal, 7(4):308, 1965.

[24] James Nicolaisen, Valentin Petrov, and Leigh Tesfatsion. Market Power and Efficiency in a Computational Electricity Market With Discriminatory Double-Auction Pricing. IEEE Transactions on Evolutionary Computation, 5(5):1-26, 2001.

[25] S.S. Oren. Design of ancillary service markets. Proceedings of the 34th International Conference on System Sciences, 2000.

[26] Simon Parsons, Juan a. Rodriguez-Aguilar, and Mark Klein. Auctions and bidding. ACM Computing Surveys, 43(2):1-59, January 2011. 
[27] J. Perloff. Microeconomics: Theory and Applications with Calculus. Addison Wesley, 2007.

[28] John G Riley. Essential Microeconomics. Cambridge University Press, 2012.

[29] Tuomas Sandholm and Subhash Suri. Optimal clearing of supply/demand curves. Lecture notes in computer science, 2002.

[30] Sebastian Stein, Enrico Gerding, Valentin Robu, and Nicholas R Jennings. A Model-Based Online Mechanism with Pre-Commitment and its Application to Electric Vehicle Charging. In Proceedings of the 11th International Conference on Autonomous Agents and Multiagent Systems (AAMAS), pages 669-676, Valencia, Spain, 2012.

[31] S. Stoft. Power Market Economics - Designing Markets For Electricity. Wiley-Interscience, 2002.

[32] J. Sun and L. Tesfatsion. Dynamic Testing of Wholesale Power Market Designs: An OpenSource Agent-Based Framework. Computational Economics, 30(3):291-327, August 2007.

[33] TenneT. The Imbalance Pricing System. Technical Report June, 2005.

[34] Leen Vandezande, Leonardo Meeus, Ronnie Belmans, Marcelo Saguan, and Jean-Michel Glachant. Well-functioning balancing markets: A prerequisite for wind power integration. Energy Policy, 38(7):3146-3154, July 2010.

[35] D.J. Veit, A. Weidlich, J. Yao, and S.S. Oren. Simulating the dynamics in two-settlement electricity markets via an agent-based approach. International Journal of Management Science and Engineering Management, 1(2):83-97, 2006.

[36] A. Virag, A. Jokic, R. Hermans, and P. van Den Bosch. Combined Bidding at Power and Ancillary Service Markets. In Proceedings of the 8th International Conference on the European Energy Market, Zagreb, 2011.

[37] P. Vytelingum, S.D. Ramchurn, T.D. Voice, Alex Rogers, and N.R. Jennings. Trading agents for the smart electricity grid. In Proc. of 9th Int. Conf. on Autonomous Agents and Multiagent Systems (AAMAS 2010), pages 897-904, 2010.

[38] P. Vytelingum, T.D. Voice, S.D. Ramchurn, A. Rogers, and N.R. Jennings. Agent-based Micro-Storage Management for the Smart Grid. In Proc. of 9th Int. Conf. on Autonomous Agents and Multiagent Systems (AAMAS 2010), pages 39-46, 2010.

[39] Bert Willems, Ina Rumiantseva, and Hannes Weigt. Cournot versus Supply Functions: What does the data tell us? Energy Economics, 31(1):38-47, 2009.

\section{Author biographies}

Nicolas Höning is a PhD student at CWI in the Intelligent Systems research group. He focuses on market-based approaches to tackle the uncertainty for decentralised players in the domain of smart grids and energy systems. Nicolas received a Bachelor's degree in cognitive science from the University of Osnabrück and a Master's degree in artificial intelligence from the Vrije Universiteit Amsterdam.

Han La Poutré is leader of the Intelligent Systems research group at CWI and full professor at Delft University of Technology, at the Department of Electrical Sustainable Energy (for one day a week). He focuses on adaptive decision making in multi-actor and uncertain environments, especially for smart energy systems. This comprises computational intelligence techniques and decentralized paradigms, like multi-agent systems and non-cooperative (economic) games. Han received his $\mathrm{PhD}$ degree in computer science from Utrecht University. 